C2016, Elsevier. Licensed under the Creative Commons Attribution-NonCommercialNoDerivatives 4.0 International http://creativecommons.org/about/downloads 


\title{
Resilient Supply Chain Network Design under Competition: A Case Study
}

\author{
Shabnam Rezapour ${ }^{1}$, Reza Zanjirani Farahani ${ }^{2 *}$, Morteza Pourakbar ${ }^{3}$ \\ (1) The School of Industrial and System Engineering, The University of Oklahoma, Norman, Oklahoma, USA \\ (2) Department of Management, Kingston Business School, Kingston University, UK \\ (3) Rotterdam School of Management, Erasmus University Rotterdam, Rotterdam, The Netherlands
}

\begin{abstract}
This research, motivated by a real-life case study in a highly competitive automobile supply chain, experimentally studies the impact of disruption on the competitiveness of supply chains. The studied supply chain faces two major risks: disruption of suppliers and tough competition from competitors. Any disruption in upstream level of the supply chain leads to an inability to meet demand downstream and causes market share to be lost to the competitors. For such a setting, a resilient topology is redesigned that can recover from and react quickly to any disruptive incidents. To this aim, we speculate there are three policies that can be used to mitigate the disruption risk, namely keeping emergency stock at the retailers, reserving back-up capacity at the suppliers, and multiple-sourcing. The problem is addressed using a mixed integer non-linear model to find the most profitable network and mitigation policies. We design a piecewise linear method to solve the model. Based on the data extracted from an automotive supply chain, practical insights of the research are extracted in a controlled experiment. Our analysis suggests that implementing risk mitigation policies not only work to the advantage of the supply chain by sustaining and improving its market share but also benefit customers by stabilizing retail prices in the market. Using the case study, we analyze the contribution of each risk strategy in stabilizing the supply chain's profit, market share, and retail price. Our analysis reveals that downstream "emergency stock" is the most preferable risk mitigation strategy if suppliers are unreliable.
\end{abstract}

Keywords: Supply chain management; resilient supply chain; disruption; competition; automotive industry.

\footnotetext{
* Corresponding author: Fax: +44 0208417 7026; Tel: +44 0208517 5098; Email: $\underline{\text { R.Zanjiranifarahani@kingston.ac.uk }}$
} 


\section{INTRODUCTION}

On March 17, 2000, a line of thunderstorms at Albuquerque, New Mexico caused some fluctuations in the electricity system that led to a fire at a Philips semiconductor manufacturing plant. This plant was the only supplier of chips to the two giant cell phone manufacturers Nokia and Ericsson. Consequently, Philips had to halt its chip production at this plant. The reactions of the two companies to this event were markedly different. Nokia reacted promptly by reserving capacity in other plants worldwide and procuring some commodity chips. However, Ericsson woke up about six weeks later when there were hardly any alternative sources left in the market and therefore bore the brunt of the disruption. This disruption led the company to lose a huge share of the market, mainly to Nokia, such that in 2001 Ericsson announced its retreat from the phone handset market (Sheffi, 2007). With the globalization of the supply chains (SCs), they have become more vulnerable to disruptive events. For example as an aftermath of the earthquake in Japan in March 2011, 80\% of the automobile plant in Japan suspended production. Nissan Motor Company Ltd was perceived to have most suffered from the disaster compared to its competitors. Despite this devastation, Nissan's recovery was remarkably better than the competitors. During the next six months after disaster, Nissan's production in Japan decreased by only $3.8 \%$ whereas the industry decrease was $24.8 \%$. More interestingly, Nissan could benefit from this incident and increased the production by the end of 2011 by $9.3 \%$ while the industry-wide production indicated a decrease of 9.3\% (Schmidt and Simchi-Levi, 2013).

What is very apparent from these cases is that in the markets that are highly competitive an enterprise that fails to sustain its operations in the face of disruption is very likely to be taken over by its competitors. The focal question entailed by these examples is how a supply chain (SC) can be designed to be able to react to adverse incidents in a timely manner and not to lose but even benefit from the incidents by consolidating and increasing the market share similar to what Nokia and Nissan could achieve. One answer suggested in the literature is applying the concept of resilient SCs (Sheffi, 2007). Resilience for companies, measures their ability to quickly return to their normal performance level - i.e., production, services and fill rate - after disruption. One way to achieve resilience is through creating redundancy (Sheffi, 2007). The standard use of redundancy includes holding safety and emergency stock of material and finished goods, the deliberate use of multiple suppliers and low capacity utilization rates to hedge against disruptions.

Hendricks and Singhal (2005) report that SC glitches and disruptions can lead to both short- and long-term loss in sales and market share. The major harm to the company after a disruption comes not from the direct damages to facilities but in the market share lost to the competitors. It is because SC disruptions could prevent a firm from capitalizing on strong market demand due to unavailability of products and as a consequence the market share is lost to the competitors. Despite the significance of SC network design (SCND) on the aftermath of the disruptions when there is competition in the market, a huge body of the literature concerns monopoly situations with a guaranteed demand in which the competitors and their market shares have effectively been ignored. One of the strongest motivations behind this research is to address the SCND problem when it is subject to unforeseen disruptions and SC operates in a competitive environment. Inspired by a real-life case from automotive industry, some of the main questions that we address in this study are as follows: How SCs can build in redundancy at the design stage in order to create resilience? How can policies such as keeping extra emergency stocks, having back-up suppliers and anticipating the need for reserve capacity enable SCs to 
hedge against SCs' market share loss and retail price fluctuations? Moreover, considering the extremely competitive nature of today's SCs, we investigate how these mitigation policies might assist SCs to maintain their market shares when facing with disruptions. Implementing these policies comes at a cost, so the other pertinent issue that we investigate is whether the benefits to be gained outweigh the losses. Using a controlled experiment, we also address the contribution of various risk mitigation strategies to the SC profit, stability of price and market shares. Our results show that, even though such policies might be considered costly and place an extra burden on SCs, once implemented they will have significant benefits if a disruption occurs. Moreover, in a competitive market, they prevent there being an under-supply of products and they therefore lead to price reductions for consumers. Moreover, this research shows the major harm to SCs exposed to disasters comes not from the direct damages caused by the disruption to facilities but in the market share lost to competitors. This paper is organized as follows: Section 2 presents an overview of several research streams in the literature pertinent to our work. Section 3 introduces the case study as the core of the paper and describes the challenges faced by the company and issues to be addressed by the model. Section 4 explains formulation of the problem and suggests a solution approach. Section 5 discusses experimental analyses based on a case study; it also includes insights for practitioners under different scenarios. Finally, section 6 concludes the research.

\section{LITERATURE REVIEW}

The most relevant stream of literature to this study is SC risk management. The main focus of this stream is to address, manage and respond to different sources of uncertainty existing in a SC. It is argued that the nature of uncertainty might be different but can be categorized into two broad groups, namely fluctuation and disruption. Fluctuation is a kind of uncertainty that includes expected, small and frequent variations such as fluctuations in market demand (Schütz et al., 2009), in price of raw materials, energy and labor (Sharma and Banerjee, 2013), in both future demand and cost (Tian and Yue, 2014), in the amount of nonconforming products violating certain standards (Khan et al., 2014), in the amount of damaged products or delays in the transportation system (Lewis, 2013). Fluctuations usually impact the operational-level decisions throughout the SC network.

Disruption can involve the kind of uncertainty that includes unexpected, huge and infrequent variations such as unavailability of facilities due to bankruptcy, fire, labor strikes, floods, earthquakes, etc. (Kleindorfer and Saad, 2005; Chopra et al., 2007) and breakdown in transportation due to extreme weather conditions, closure of national borders due to wars, sanctions, terrorist attacks or strikes at ports (Wilson, 2007). Potential disruptions should be considered in strategic-level decisions dealing with the selection of SC partners and the design of the SC network structure.

Several risk mitigation strategies for lessening the impact of disruption are explored in the literature. Tang and Tomlin (2008) argue that mitigation strategies create flexibility in SCs that leads to a better performance. These mitigation strategies are discussed and implemented either at a strategic or an operational level. A popular strategy in both practice and literature is to anticipate back-up facilities such that if there is disruption to a critical facility, the back-up facilities can then be utilized to maintain the production process (Chopra et al., 2007; Dada et al., 2007). Another approach is to create redundant or extra reserve capacity in some facilities to be used if disruption occurs (Chopra et al., 2007; Chopra and Sodhi, 2004). Moreover, at the operational level, other strategies have been proposed as a way of neutralizing the risk of fluctuations such as keeping safety 
stock in the inventory system (Shen and Daskin, 2005), production postponement and downward substitution (Lin and Wang, 2011). Another popular strategy is sourcing from multiple suppliers in case of supplier unreliability that can be in the form of back-up facilities or extra reserve capacity (Tomlin, 2006; Feng and Shi, 2011).

Some researchers address the problem of designing a SC in the presence of risk and uncertainty. Goh et al. (2007) provide a solution to a stochastic model of the multi-stage global SCND including location and distribution decisions. The research takes into account supply, demand, exchange, and disruption risk. Qi et al. (2010) study an integrated SC design under random supply disruption. The model determines the locations of retailers and the allocation of customers to retailers such that the total cost is minimized. Klibi and Martel (2012) study various models applicable to the design of resilient SC network for both location and distribution under uncertainty. Baghalian et al. (2013) develop a stochastic programming formulation to model a multiproduct SCN with both demand and supply uncertainties. Huang and Goetschalckx (2014) define the strategic robust SC design as the set of all Pareto-optimal configurations considering efficiency and risk simultaneously, where the risk is measured by the standard deviation of the efficiency. Interested readers may refer to Klibi et al. (2010) and Farahani et al. (2014) to learn about the literature of SCND under uncertainty and the models used to address these problems.

So far, the existing literature on SCND for risk management only considers monopoly situations. This basically results in ignoring the impact of SC disruptions on inability to meet the demand in the market, which could possibly have the highest negative impact on SCs in the event of disruptions. What differentiates this paper from the rest of the literature on SCND is that, we analyze the impact of incorporating the risk management strategies on the competitive capabilities of SCs such as captureable market share, profit-ability and retail price.

One other relevant topic in the literature is the study of ripple effect in supply chains (Ivanov et al., 2014a, 2014b). Ripple effect is defined as studying the impact of a disruption on supply chain performance and disruption-based scope of changes in the supply structures and flows (Ivanov et al. 2014b). In this research we do not explicitly address the ripple effect. However, it is implicitly related to this effect as it captures the impact of disruption at any stage within the supply chain on the price of the product and market share lost to the competitors. In other words, we study how the impact of disruption ripples through the supply chain and impacts the downstream side of the chain.

The other relevant stream of literature to this study is the competition between SCs. Boyaci and Gallego (2004) have investigated the problem of chain-to-chain competition on customer service. They show that a coordinated strategy is dominant and customers are the only beneficiaries under such strategy. Zhang (2005) studies a network consisting of multiple heterogeneous SCs competing on the market share and characterizes optimal market share in equilibrium. Xiao and Yang (2006) develop a price-service competition model of two SCs under demand uncertainty. They analyze the effects of the retailers' risk sensitivity on the players' optimal strategies. Ha and Tong (2008) study contracting mechanisms that can lead to information sharing between two competing SCs. This stream of the literature addresses the competition between the SC and rivals while the network structure of the SC is assumed to be predetermined. However, in this study, given the significant impact of SC structure on the price and market shares, the considered competitive SCND problem goes beyond 
a competitive SC planning. We present a model that optimizes the design of the SC network including the number, location and capacity decisions for different entities within the SC. Furthermore, we also account for the possibility of supply disruption and, accordingly, consider risk mitigation strategies by creating an optimal level of redundancy in the network. The redundancy is created through emergency stock, reserve capacity and backup suppliers. The model allows us to study the impact of such design on cost efficiency and market share under supply uncertainty.

\section{PROBLEM DEFINITION}

The problem studied in this paper is based on a real-world case. In this section, the case problem is briefly explained. Then, the problem is defined in a form which is more appropriate for modeling.

\subsection{Case study: SMAC company}

The case problem is in the automotive industry but the names of the companies involved are anonymized. SMAC is one of the largest axial parts and reverse idler gear shaft producers in the region having some automotive manufacturers as customers of those parts. To produce these items, SMAC needs to procure two components, CK45 steel and barbed pin. AKC is the sole supplier of barbed pin but CK45 steel can be sourced from YIC as a national supplier and also from overseas suppliers ${ }^{1}$. The abundance of shaft providers in the market and the similar quality of their products mean that these rival companies compete in the market on the basis of product price.

At present, disruption and delay in procuring CK45 steel from suppliers is one of the most crucial challenges for SMAC, forcing the company to redesign its network structure to mitigate the negative effects of this uncertainty. It operates in a very competitive environment and any disruption has a drastic effect on its market share. To guard against the effects of future disruptions, SMAC wants to redesign its SC and for this it needs to make the following decisions: I) which suppliers should be selected (opportunity for multiplesourcing)?; II) at each selected supplier, how much production capacity should be assigned to SMAC? (opportunity for extra capacity); and III) how should the demand for CK45 steel be assigned to the suppliers in the event of disruption? Moreover, it is also planned to keep emergency stock in the SC network, thus requiring two further decisions: I) where these stocks should be located? and II) how many products should be stored in each place? Reverse idler gear shaft is a cheap product with Make-To-Stock (MTS) production strategy. Therefore, it is a viable option to recommend SMAC to keep emergency stocks in its retailers' locations.

In the case problem, we only focus on possibility of disruption in SMAC's suppliers. However, the models developed in Section 4 could be easily extended to handle disruptions in all facilities of SCs (including manufacturers, retailers and transportation links in between). In the models, we define some scenarios corresponding to possible disruptions. Each scenario is shown by a subset of SC's paths that are out-of-use in the event of disruption because at least one of their passing facilities (such as supplier, manufacturer, or retailer) or connecting links is disrupted. This modeling approach allows us to incorporate any possible disruption in the network.

\footnotetext{
${ }^{1}$ Having one common and large supplier/ manufacturer is one of characteristics of this case. While in some other research works such as Zhao (2008), Ha and Tong (2008) and Narayanan and Raman (2005) existence of only one supplier is presumed, the research methodology can simply be generalized to more than one supplier.
} 


\subsection{Problem description}

In this paper, we consider a SC producing and supplying a product to known markets. There are several other rivals in the markets supplying the same or different but substitutable products. The SC is competing with those rivals for market share. The product's retail price in the markets depends on the total product quantity supplied by rivals to the markets. The potential demand in the markets is a function of the product's retail price. Therefore, each rival directly selects its product supply quantity and indirectly selects the product's retail price and potential demand in the markets such that its profit is maximized. Assuming that all rivals make decisions on their production quantities concurrently, we are able to find Nash equilibrium. In this paper, competition among rivals affects the following items:

- Competition determines "amount of products that should be manufactured and supplied by each rival to the markets - so-called, its market share - and potential demand of product in the markets". Potential demand in markets is usually assumed fixed in the literature.

- Competition determines the "product's retail price" in the markets as the product's retail price depends on the total product supply to the markets. As expected, lower product supply to the markets after disruption leads to a higher retail price. The product price is usually considered an exogenous parameter in the previous studies in the literature.

Therefore, our modelling has two parts: a) modelling competition among rivals to determine the equilibrium retail price, potential markets' demands, and market shares (“Competition" part in Figure 1) that will be explained in Section 3.2; and b) developing a model to select the best risk mitigation strategies and designing the most profitable network for the SC ("Network Design" module in Figure 1) that will be done in Section 3.3.

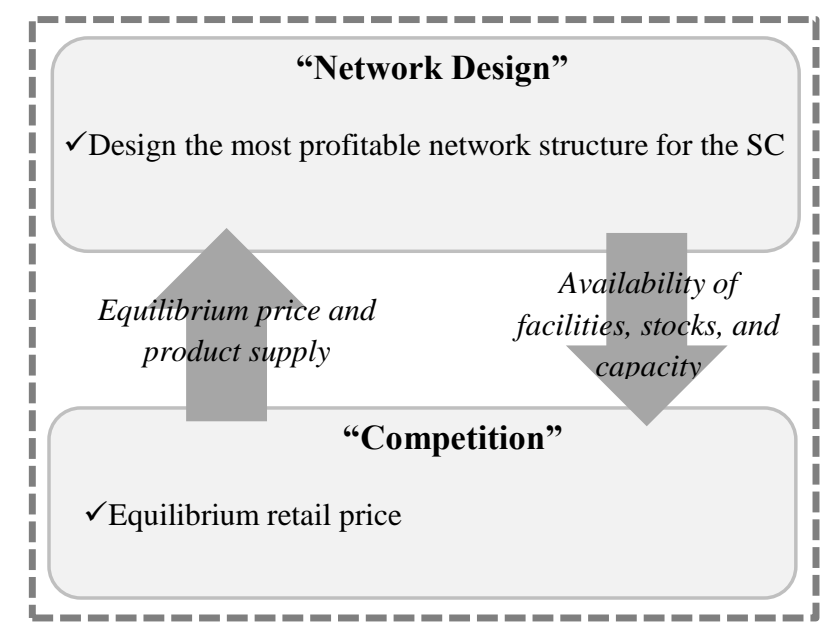

Figure 1. Two main components in the research problem.

Network design and competition problems which are solved simultaneously are not independent; some important interactions between these two parts are as follows:

- Information flow from “Network Design” module to “Competition” module: Availability of facilities, stocks, and capacity is determined by "Network Design" module and used by "Competition" module to determine the equilibrium product supply and retail price (see Figure 1).

- Information flow from “Competition" module to "Network Design" module: The equilibrium product supply and retail price determined by "Competition" module is used by "Network Design" 
module to compute its capture-able income and profit (see Figure 1).

In this paper, we study the importance of risk mitigation strategies on redesigning SCs to improve their competitive advantages. We want to determine what is the contribution of incorporating risk mitigation strategies in stabilizing 1) the SC's profit; 2) its market share; and 3) retail price.

\section{MODELING AND SOLUTION}

Without loss of generality, we assume that this acyclic ${ }^{2}$ SC consists of multiple suppliers, one manufacturer and multiple retailers. Each retailer is for servicing one of the target markets. The set of all available suppliers which can be used by the SC is denoted by $I, I=\{i|i=1,2, \ldots| I \mid$,$\} . The manufacturer sources components$ from these suppliers and manufactures the final products. Then the products are shipped to retailers to serve markets. $M$ is used to denote the set of available target markets to which products can be supplied, $M=\{m \mid$ $m=1,2, \ldots,|M|\}$.

There are several rivals in the target markets offering the same product and competing to capture a higher market share. The set of existing rivals in the market $m$ is denoted by $R^{(m)}$. We assume that the demand of each potential market is elastic and decreasing in the retail price of the product. The quantity sold in the market $m$, $Q_{m}$, is defined as, $Q_{m}=\sum_{r=1}^{\left|R^{m}\right|} \bar{q}_{r}+q_{m}$ where it is the sum of the quantities supplied by the rivals, $\bar{q}_{r}(\forall r \in$ $\left.R^{(m)}\right)$, and the quantity supplied by the SC, $q_{m}$. Moreover, the price of the product in the market $m$ is a linear function of its supply quantity, $Q_{m}$, shown as $p_{m}=a_{m}-b_{m} Q_{m}$. In this relation, $a_{m}$ is the maximum price at which the quantity of the product sold in market $m$ is positive and $b_{m}$ is the price sensitivity parameter in market $m$.

Suppliers might be subject to disruptions. In order to model disruptions, we define a path $t_{i m}$ that starts from supplier $i(\forall i \in I)$, goes via the manufacturer, and delivers the goods to the retailer of market $m$ ( $\forall m \in$ $M)$. This allows us to represent the SC's potential network structure as a set of potential supply paths. Selecting the best network for the SC can be abstracted as selecting the best subset of these potential paths. For example, let us assume a SC (as depicted in Figure 2) characterized by six potential supply paths $T=\left\{t_{11}, t_{12}, t_{21}, t_{22}, t_{31}, t_{32}\right\}$. The developed model will select the optimal subset of paths; let us assume it is the subset $\left\{t_{11}, t_{12}, t_{21}, t_{22}\right\}$. This means that the best network structure includes suppliers 1 and 2 in the first echelon and target markets 1 and 2 in the last echelon.

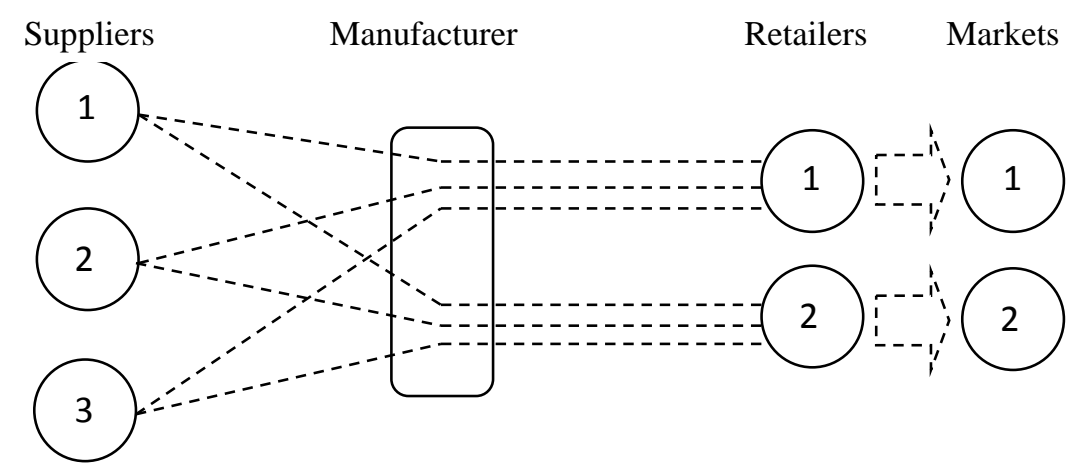

Figure 2. Potential paths in a sample SC.

\footnotetext{
${ }^{2}$ Such acyclic supply chain networks are common in the automobile and apparel industries (Majumder and Srinivasan, 2008).
} 
The concept of path helps us model any disruption regardless of its reason. The effect of any disruption in the production facilities, suppliers or in the transportation (i.e., connecting) links can be represented by a subset of potential paths which are operative. For example, in the sample SC of Figure 2 assume that disruption is possible in Supplier 2 and the connecting link of the manufacturer and Retailer 2 ( $\operatorname{man} \rightarrow r 2)$ with $P r_{1}$ and $\mathrm{Pr}_{2}$ probabilities. In this case, four scenarios define the availabilities of this SC, $S=\left\{s_{1}, s_{2}, s_{3}, s_{4}\right\}$. In $s_{1}$, representing the unavailability of Supplier 2, only $\left\{t_{11}, t_{12}, t_{31}, t_{32}\right\}$ paths are available. The occurrence probability of this scenario, $P r_{s_{1}}$, is equal to $P r_{1}\left(1-P r_{2}\right)$. Scenario $s_{2}$ represents the unavailability of link (man $\rightarrow r 1$ ) by considering $\left\{t_{11}, t_{21}, t_{31}\right\}$ as the available paths with occurrence probability, $P r_{S_{2}}$, equal to $P r_{2}\left(1-P r_{1}\right)$. In $s_{3}$ only available paths are $\left\{t_{11}, t_{31}\right\}$ with $P r_{s_{3}}=P r_{1} P r_{2} . s_{4}$ represents normal conditions when all paths are operative with occurrence probability equal to $P r_{S_{4}}=1-P r_{1}\left(1-P r_{2}\right)-P r_{2}\left(1-P r_{1}\right)-$ $\operatorname{Pr}_{1} \operatorname{Pr}_{2}$.

We assume that availability of an unreliable facility in a sale period has a binomial distribution with a mean that is equal to $\theta=\frac{N u m_{D}}{N u m_{T}}\left(N u m_{T}\right.$ shows the total number of former periods for which historical data is available and $\mathrm{Num}_{D}$ shows the number of former periods in which the facility is not available).

Therefore, if there is a SC with $U N=\{u n\}$ unreliable facilities (production facilities or transportation links), the scenario set, $S$, for that chain would have $2^{|U N|}=\left(\begin{array}{c}U N \\ 0\end{array}\right)+\left(\begin{array}{c}U N \\ 1\end{array}\right)+\left(\begin{array}{c}U N \\ 2\end{array}\right)+\cdots+\left(\begin{array}{c}U N \\ U N-1\end{array}\right)+$ $\left(\begin{array}{l}U N \\ U N\end{array}\right)$ members. The first term of this series, $\left(\begin{array}{c}U N \\ 0\end{array}\right)$, shows the normal condition without any disrupted facility. The probability of this scenario is $\prod_{\forall u n \in U N}\left(1-P r_{u n}\right)$. The second term of this series, $\left(\begin{array}{c}U N \\ 1\end{array}\right)$, includes all scenarios with only one disrupted facility. The probability of the scenario in which facility $u n \in U N$ is disrupted is equal to $P r_{u n} \cdot \prod_{\substack{\forall u n \in U N \\ u n \neq u n}}\left(1-P r_{u \dot{n}}\right)$. The third term of this series, $\left(\begin{array}{c}U N \\ 2\end{array}\right)$, includes all scenarios with two disrupted facilities. The probability of the scenario in which facilities $u n$ and $u n \in U N$ are disrupted is equal to $P r_{u n} \cdot P r_{u n} \cdot \prod_{\substack{\forall u n^{\prime \prime} \in U N \\ u n^{\prime \prime} \neq u n \text { and } u n}}\left(1-P r_{u n^{\prime \prime}}\right)$. Finally, the last term, $\left(\begin{array}{l}U N \\ U N\end{array}\right)$, shows the scenario in which all unreliable facilities are disrupted. The probability of this scenario is $\prod_{\forall u n \in U N} P r_{u n}$.

The set of all feasible paths in the SC is denoted by $T, T=\left\{t_{i m} \mid i \in I\right.$ and $\left.m \in M\right\}$. Any disruptions in the facilities or the links on the path would disrupt the entire path. We consider all the possible disruptions in the $\mathrm{SC}$ and associate a scenario to each combination of them. The set of all scenarios is denoted by $S, S=\{s \mid s=1$, $2, \ldots,|S|\}$. The probability that each scenario will occur is denoted by $\operatorname{Pr}_{S}$, s $\in \mathrm{S}$.

Three possible risk mitigation strategies are considered to neutralize the negative effects of disruptions. These strategies are reserving extra production capacity at the suppliers, multiple-souring, and keeping extra inventory or 'emergency stock' at the retailers. The main decisions addressed by the model can be categorized as strategic and tactical. Strategic decisions include which suppliers should be selected and what their optimal capacity should be. Moreover, the model should select the optimal markets to which the products are delivered so that the total profit is maximized. Tactical decisions deal with questions such as which routes should be used to meet the demand of each market and what is the share of each route in the total sales of each market? Should there be any emergency stocks held at retailers and what are the optimal levels? What are the optimal market 
shares for the SC and its rivals, and what is the best selling price for the product in the market? A hierarchical decision-making process, which addresses first strategic and then tactical decisions, leads to sub-optimal solutions. This is because the efficiency of tactical decisions is affected by the network structure. The profit that can be achieved at the tactical stage depends on its network structure decided at the strategic level.

Here we consider a single-sale period SCND problem. Multi-sale period production and storage planning is usually used for products for which the demand realization pattern significantly changes inside the planning period such as products with seasonal demands. Since in our case problem such a significant demand fluctuation is not expected, we consider a single-sale period as a representation of the whole planning period. When a link or a facility of a path is disrupted, this means the products moving on that path cannot be delivered on time to the retailer and it is very unlikely that disruption lasts more than a single-sale period.

We use path concept based on cut-set theory -which is originated from reliability theory (Baghalian et al. (2013)- to formulate the models. Each path starts from a facility in the first echelon (a supplier in the case problem), passes through a facility in intermediate echelons (the manufacturer in the case problem), and finally ends at a facility in the last echelon (a retailer in the case problem). Therefore, the SC is represented as a set of paths. By changing the network structure of the SC, we only need to redefine "path" and update set. Therefore, the models of this paper are not restricted to SCs with a specific network structure and they can be applied for any SCs with any network structure.

In the rest of this section, we first model a non-resilient network in which it is assumed that all facilities are always available. We then explore the possibility that some of the facilities will face disruption, and propose a resilient model using some risk mitigation strategies to hedge against such disruptions.

\subsection{Non-resilient and competitive SCND}

In this case, we ignore the possibility that there will be disruptions in the network. Therefore, there is only one scenario called "normal conditions", $S=\{n\}$. With this assumption, we model the problem such that the total profit of the $\mathrm{SC}$ is maximized. The problem formulation is expressed as follows.

\section{Sets and indices}

I: Set of all available suppliers which can be used by SC, $I=\{i|i=1,2, \ldots| I \mid$,$\} ;$

$M$ : Set of target markets for supplying products of SC, $M=\{m|m=1,2, \ldots| M \mid$,$\} ;$

$T$ : Set of all potential paths in the potential network structure of the SC, $T=\left\{t_{i m} \mid i \in I\right.$ and $\left.m \in M\right\}$;

$T^{(i)}$ : Subset of paths of $T$ started from supplier $i, T^{(i)} \subset T$;

$T^{(m)}$ : Subset of paths of $T$ ended at market $m, T^{(m)} \subset T$;

$S:$ Set of all possible scenarios;

$T_{(s)}$ : Subset of paths of $T$ which are usable in scenario $s, T_{(s)} \subset T$;

$R^{(m)}$ : Subset of existing rivals in market $m, \hat{t} \in R^{(m)}$;

$K_{t}^{s}$ : Number of linear parts included in the linearized equilibrium profit and flow functions of path $t$ in scenario $s$.

\section{Variables}

$v_{i}: 1$ if supplier $i$ is selected by the $\mathrm{SC}, 0$ otherwise $(\forall i \in I)$; 
$\bar{v}_{i}$ : Production capacity of supplier $i$ assigned to the $\mathrm{SC}(\forall i \in I)$;

$w_{m}: 1$ if target market $m$ is selected to supply products in the SC; 0 otherwise $(\forall m \in M)$;

$x_{t}^{S}$ : Percentage of the SC's capturable demand in market $m$ that is supplied through path $t$ in scenario $s(\forall m \in$ $M, \forall t \in T^{m}$, and $\left.\forall s \in S\right)$. Notice that if $\left|T^{(m)}\right|=1$, then $x_{t}^{s}\left(t \in T^{(m)}\right)$ changes to a binary variable;

$y_{t}^{s}: 1$ if inventory is kept in the corresponding retailer of path $t$ in its inaccessibility scenarios; 0 otherwise $\left(\forall t \in T_{(s)}, \forall s \in S\right)$;

$z_{t}$ : Amount of path $t^{\prime}$ s product kept in its ending retailer for being used in case of disruption $(\forall m \in M)$;

$p_{m}$ : Price of the SC's product in market $m(\forall m \in M)$;

$q_{m}$ : Amount of product supplied by the SC to market $m(\forall m \in M)$;

$\bar{q}_{r}$ : Amount of product supplied by existing rival $r$ in market $m\left(r \in R^{(m)}\right)$;

$\alpha_{t, k}^{S}: 1$ if variable $x_{t}^{S}$ accepts a value in the linear piece $k$ of its piecewise linearized profit function; 0 otherwise $\left(\forall t \in T, k=1,2, \ldots, K_{t}^{S}\right)$. Notice that $\sum_{k=1}^{K_{t}^{S}} \alpha_{t, k}^{s}=w_{m}\left(\forall t \in T^{(m)}, \forall m \in M\right) ;$

$\hat{\alpha}_{t, k}^{S}: 1$ if variable $x_{t}^{S}$ accepts a value in the linear piece $k$ of its piecewise linearized flow function; 0 otherwise $\left(\forall t \in T, k=1,2, \ldots, K_{t}^{S}\right)$. Notice that $\sum_{k=1}^{K_{t}^{S}} \dot{\alpha}_{t, k}^{S}=w_{m}\left(\forall t \in T^{(m)}, \forall m \in M\right) ;$

\section{Parameters}

$k_{i}$ : Cost of unit capacity of supplier $i$ assigned to the SC $(\forall i \in I)$;

$B_{m}$ : Fixed cost of locating a retailer in market $m(\forall m \in M)$;

$h_{m}$ : Unit holding cost in retailer $m$ along each period $(\forall m \in M)$;

$c_{i}$ : Cost of producing and distributing unit along the path $t(i \rightarrow m), c_{t}=a^{\prime}{ }_{i}$ (unit production cost in the SC's

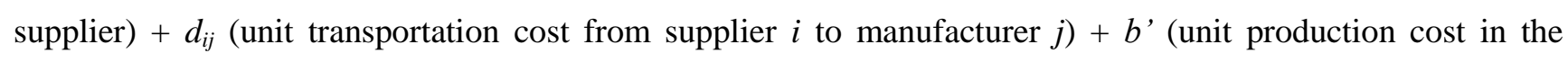
manufacturer) $+d_{m}$ (unit transportation cost from the manufacturer to retailer $m$ ) $+c_{m}^{\prime}$ (unit handling cost in retailer of market $m)(\forall t \in T)$;

$c_{r}$ : Cost of producing and distributing product unit by rival $r$ in market $m\left(r \in R^{(m)}, \forall m \in M\right)$;

$e_{t}^{s}: 1$ if path $t$ is usable in scenario $s ; 0$ otherwise $(\forall t \in T, \forall s \in S)$;

$P r_{s}$ : Probability of scenario $s(\forall s \in S)$;

$a_{m}$ : Maximum price at which the quantity of the product sold in market $m$ is positive $(\forall m \in M)$;

$b_{m}$ : Price sensitivity parameter in market $m$;

$\theta_{t, k}^{S}$ : Constant term of $k^{\text {th }}$ linear piece in the linearized equilibrium profit function of path $t$ in scenario $s$ $\left(\forall t \in T, \forall s \in S, k: 1,2, \ldots, K_{t}^{S}\right)$;

$\vartheta_{t, k}^{S}$ : Coefficient of $k^{\text {th }}$ linear piece in the linearized equilibrium profit function of path $t$ in scenario $s(\forall t \in$ $\left.T, \forall s \in S, k: 1,2, \ldots, K_{t}^{s}\right)$;

$\delta_{t, k}^{S}$ : Upper bound of $k^{\text {th }}$ linear piece in the linearized equilibrium profit function of path $t$ in scenario $s$ $\left(\forall t \in T, \forall s \in S, k: 1,2, \ldots, K_{t}^{S}-1\right)$;

$\hat{\theta}_{t, k}^{s}$ : Constant term of $k^{\text {th }}$ linear piece in the linearized equilibrium flow function of path $t$ in scenario $s$ $\left(\forall t \in T, \forall s \in S, k: 1,2, \ldots, K_{t}^{S}\right)$;

$\hat{\vartheta}_{t, k}^{s}$ : Coefficient of $k^{\text {th }}$ linear piece in the linearized equilibrium flow function of path $t$ in scenario $s(\forall t \in$ 
$\left.T, \forall s \in S, k: 1,2, \ldots, K_{t}^{s}\right)$;

$\delta_{t, k}^{S}$ : Upper bound of $k^{\text {th }}$ linear piece in the linearized equilibrium flow function of path $t$ in scenario $s(\forall t \in$ $\left.T, \forall s \in S, k: 1,2, \ldots, K_{t}^{s}-1\right)$;

$$
\operatorname{Max} \quad Z=\sum_{m=1}^{|M|} \sum_{t=1}^{\left|T^{(m)}\right|}\left(\tilde{p}_{m}-c_{t}\right) x_{t}^{n} \tilde{q}_{m}-\sum_{i=1}^{|I|}\left(k_{i} \bar{v}_{i}\right)-\sum_{m=1}^{|M|}\left(B_{m} w_{m}\right)-\sum_{i=1}^{|I|}\left(F_{i} v_{i}\right)
$$

Subject to:

$$
\begin{array}{ll}
\sum_{t=1}^{\left|T^{(m)}\right|} x_{t}^{n}=w_{m} & (\forall m \in M) \\
\sum_{t=1}^{\left|T^{(i)}\right|} x_{t}^{n} q_{m_{\mid m \in t}} \leq \bar{v}_{i} & (\forall i \in I) \\
x_{t}^{n} \leq v_{i} & \left(\forall t \in T^{(i)}, \forall i \in I\right) \\
x_{t}^{n} \leq w_{m} & \left(\forall t \in T^{(m)}, \forall m \in M\right) \\
0 \leq x_{t}^{n} \leq 1 & (\forall t \in T) \\
\bar{v}_{i} \geq 0 \text { and } w_{m} \text { and } v_{i} \in\{0,1\} & (\forall m \in M, \forall i \in I)
\end{array}
$$

The objective function (1) represents the SC's total profit, which is the difference between the revenue generated by selling products in different markets and the total cost. $x_{t}^{n}$ is the percentage of the SC's demand in market $m$ that is supplied via path $t . c_{t}$ is the cost of producing and distributing product unit along path $t$. Therefore, the total profit of the SC in market $m$ through path $t\left(\forall t \in T^{(m)}\right)$ is equal to the multiplication of that path's equilibrium profit in that market, $\tilde{p}_{m}-c_{t}$, and the equilibrium amount of product sold via that path, $x_{t}^{n} \tilde{q}_{m}$. The first term calculates the SC's total profit as the sum of $\left(\tilde{p}_{m}-c_{t}\right) x_{t}^{n} \tilde{q}_{m}$ over all available paths in all markets. The second term formulates the capacity cost of suppliers where $k_{i}$ is the unit capacity cost and $\bar{v}_{i}$ represents total reserved capacity in supplier $i(i \in I)$. The third term in (1) calculates the retailers' location cost where $B_{m}$ is the fixed cost of locating a retailer in market $m$ and $w_{m}$ is a binary decision variable that is set to 1 if market $m$ is selected to supply products and to 0 otherwise. The fourth term in (1) calculates the selected suppliers' investment cost where $F_{i}$ is the fixed investment cost of supplier $i$ and $v_{i}$ is a binary decision variable that is set to 1 if supplier $i$ is selected and to 0 otherwise.

Constraint (2) ensures that the paths leading to each market are activated only if there is a retailer located in that market. $T^{(m)}$ is the subset of $T$ including all the paths that end at market $m$, i.e. $T^{(m)}=\left\{t_{i m} \mid \forall i \in I\right\}$. Constraint (3) dictates that the amount of flow from each supplier should be lower than the reserved capacity of that supplier, $\bar{v}_{i} \cdot T^{(i)}$ is the subset of $T$ including all the paths originating from supplier $i$, i.e. $T^{(i)}=$ $\left\{t_{i m} \mid \forall m \in M\right\}$. Constraints (4-5) ensure that facilities are located along the active paths of the SC.

In Model (1-7), the equilibrium prices, $\tilde{p}_{m}(\forall m \in M)$, and the equilibrium product supplies, $\tilde{q}_{m}(\forall m \in$ $M)$, are not exogenous parameters and depend on the competition among rivals. In Section 3.2, we model competition among rivals in the markets to figure out how equilibrium prices and product supplies are functions of the SC's operational and strategic level decisions.

\subsection{Equilibrium flow and price in competitive markets}

In this section, we determine the equilibrium supply, $\tilde{q}_{m}(\forall m \in M)$, and retail price, $\tilde{p}_{m}(\forall m \in M)$, used in equation (1) to compute the SC's revenue. Each rival in the market chooses the quantity of products to produce such that its profit is maximized. As explained before in $p_{m}=a_{m}-b_{m} Q_{m}$ equation, the total amount of products delivered determines the retail price in the market, shared by all rivals. Therefore, the profit of the SC in market $m$ in the normal conditions, $s=n$, would be as follows: 


$$
\pi_{m}^{n}=\left(a_{m}-b_{m}\left(\sum_{r=1}^{\left|R^{(m)}\right|} \bar{q}_{r}+q_{m}\right)\right)\left(q_{m}\right)-\sum_{t=1}^{\left|T^{(m)}\right|} c_{t} q_{m} x_{t}^{n}
$$

Assuming that all the market rivals make decisions concurrently on their production quantities, Nash equilibrium is used to model their competition. Results of the Nash equilibrium when the SC competes in market $m$ with the rivals are indicated in Lemma 1. The proof of Lemma 1 is presented in the Appendix A.

Lemma 1. The equilibrium supply and price of the SC in market $m$ is given by

$$
\begin{array}{ll}
\tilde{q}_{m}=\left(a_{m}-\sum_{t=1}^{\left|T^{(m)}\right|} c_{t} x_{t}^{n}\right) / b_{m}\left(1+\left|R^{(m)}\right|+w_{m}\right) & (\forall m \in M) \\
\tilde{p}_{m}=\left(a_{m}+\sum_{r=1}^{\left|R^{(m)}\right|} c_{r}+\sum_{t=1}^{\left|T^{(m)}\right|} c_{t} x_{t}^{n}\right) /\left(1+\left|R^{(m)}\right|+w_{m}\right) & (\forall m \in M)
\end{array}
$$

Now we substitute these results in the proposed mathematical model (1-7). With these substitutions, the objective function (1) changes as follows:

$$
\begin{aligned}
\operatorname{Max} & \sum_{m=1}^{|M|}\left[\sum_{t=1}^{\left|T^{(m)}\right|}\left(\frac{a_{m}+\sum_{r=1}^{\left|R^{(m)}\right|} c_{r}+\sum_{t=1}^{\left|T^{(m)}\right|} c_{t} x_{t}^{n}}{\left(1+\left|R^{(m)}\right|+w_{m}\right)}-c_{t}\right)\left(\frac{a_{m}-\sum_{t=1}^{\left|T^{(m)}\right|} c_{t} x_{t}^{n}}{b_{m}\left(1+\left|R^{(m)}\right|+w_{m}\right)}\right) x_{t}^{n}-\sum_{i=1}^{|I|}\left(k_{i} \bar{v}_{i}\right)-\right. \\
& \sum_{m=1}^{|M|}\left(B_{m} w_{m}\right)-\sum_{i=1}^{|I|}\left(F_{i} v_{i}\right)
\end{aligned}
$$

However, in equation (11), we can set $w_{m}=1$ in the denominators of both of the above fractions. It is because, in the case of $w_{m}=0, x_{t}^{n}$, the variable representing all paths ending at market $m\left(\forall t \in T^{(m)}\right)$, would be zero due to equation (2) which makes the first term of (11) equal to zero. Therefore, this term is only positive if $w_{m}=1$. The calculated equilibrium flow should also be substituted in constraint (3) of the mathematical model:

$$
\sum_{t=1}^{\left|T^{(i)}\right|} x_{t}^{n}\left(\frac{a_{m \mid m \in t}-\sum_{t=1}^{\left|T^{(m)}\right|} c_{t} x_{t}^{n}}{b_{m}\left(1+\left|R^{(m)}\right|+w_{m}\right)}\right) \leq \bar{v}_{i} \quad(\forall i \in I, m \text { is a market at which path } t \text { ends })
$$

With the same reasoning, we can set $w_{m}=1$ in the above constraint. The mathematical model of the problem in the non-resilient case would therefore be as follows:

$\operatorname{Max} \sum_{m=1}^{|M|}\left[\sum_{t=1}^{\left|T^{(m)}\right|}\left(\frac{a_{m}+\sum_{r=1}^{\left|R^{(m)}\right|} c_{r}+\sum_{t=1}^{T^{(m)} \mid} c_{t} x_{t}^{n}}{\left(2+\left|R^{(m)}\right|\right)}-c_{t}\right)\left(\frac{a_{m}-\sum_{t=1}^{\left|T^{(m)}\right|} c_{t} x_{t}^{n}}{b_{m}\left(2+\left|R^{(m)}\right|\right)}\right) x_{t}^{n}-\sum_{i=1}^{|I|}\left(a_{i} \bar{v}_{i}\right)-\sum_{m=1}^{|M|}\left(B_{m} w_{m}\right)-\right.$

$\sum_{i=1}^{|I|}\left(F_{i} v_{i}\right)$

Subject to:

$$
\sum_{t=1}^{\left|T^{(i)}\right|} x_{t}^{n}\left(\frac{a_{m}-\sum_{t=1}^{\left|T^{(m)}\right|} c_{t} x_{t}^{n}}{b_{m}\left(2+\left|R^{(m)}\right|\right)}\right) \leq \bar{v}_{i} \quad(\forall i \in I, m \text { is a market at which path } t \text { ends })
$$

Constraints (2 and 4-7): Constraints 2 and 4-7 remain unchanged.

By solving Model (13-15) and selecting the most profitable supply paths, variables $\boldsymbol{x}_{\boldsymbol{t}}^{\boldsymbol{n}}$, the equilibrium price and supply quantities are determined. Therefore, network design decisions, equilibrium supply and retail price decisions are made simultaneously by the model.

\subsection{Resilient and competitive SCND}

In this section, we develop a model of a resilient and competitive SCND. We define several scenarios 
representing disruption possibilities in the links and facilities through the subsets of potential paths that are available to produce and supply products. To determine the availability of paths in the scenarios, we define a new binary parameter $e_{t}^{S}(\forall t \in T, \forall s \in S)$ which is set to 1 if path $t$ is available in scenario $s$ and to 0 otherwise. In the rest of this section, we discuss the corollaries of incorporating risk mitigation strategies, Multiple-sourcing, reserving extra capacity and keeping emergency stock, in the mathematical model (13-15) to develop the model of designing resilient and competitive SCND.

Model adjustment 1: Variable $x_{t}^{S}$ represents the percentage of demand in the ending market of path $t$ that is assigned to this path in scenario $s(\forall t \in T, \forall s \in S)$. To incorporate "multiple-sourcing" mitigation strategy, the variable assigning flows to paths should change to $x_{t}^{s}(\forall t \in T, \forall s \in S)$ in the model. Adding the index $s$ to this variable enables the model to use different paths and their associated facilities to meet market demands in various scenarios. Facilities through the paths substituting disrupted ones in a scenario are other sources to be used in the event of disruption.

By adding "Multiple-sourcing" mitigation strategy, more facilities (such as suppliers) will be selected and consequently more investment cost will be imposed to the SC (fourth term in Equation (1) and fifth term in Equation (22) that will be explained later).

Model adjustment 2: To incorporate "Emergency Stock" mitigation strategy, two new variables should be defined in the model namely $y_{t}^{S}$ and $z_{t} \cdot y_{t}^{S}(\forall t \in T)$ decide about the paths using emergency stock strategy in scenario $s(\forall s \in S)$; and $z_{t}(\forall t \in T)$ determine the level of stock that should be kept for each path in its corresponding retailer. The following equations are required to control the values of these variables in the model:

$$
\begin{array}{ll}
x_{t}^{S} \leq e_{t}^{s}+y_{t}^{s} & (\forall t \in T, \forall s \in S) \\
y_{t}^{S} \leq 1-e_{t}^{s} & (\forall t \in T, \forall s \in S) \\
z_{t} \geq\left(\frac{a_{m}-\sum_{t=1}^{\left|T^{(m)}\right|} c_{t} x_{t}^{s}}{b_{m}\left(2+\left|R^{(m)}\right|\right)}\right) x_{t}^{S} y_{t}^{s} & (\forall t \in T, \forall s \in S)
\end{array}
$$

Based on equation (16), each path can be used to supply product in a scenario, $x_{t}^{S}>0$, either if that path is operative, $e_{t}^{S}=1$, or that path is using emergency stock strategy, $y_{t}^{s}=1$. Based on equation (17), using emergency stock is only possible in the scenarios in which the path is inoperative, $e_{t}^{S}=0$. Equation (18) assures that emergency stock kept at each path should suffice to provide the path with the assigned flow in all of the scenarios in which $y_{t}^{s}=1$.

By adding "Emergency Stock" mitigation strategy, inventory holding cost will be imposed to the SC for unused stock (second term in Equation (22) that will be explained later).

Model adjustment 3: To incorporate "Extra Reserved Capacity" mitigation strategy, the following equation should be added to the model:

$$
\sum_{t=1}^{\left|T^{(i)}\right|} x_{t}^{S}\left(1-y_{t}^{S}\right)\left(\frac{a_{m \mid m \in t}-\sum_{t=1}^{\left|T^{(m)}\right|} c_{t} x_{t}^{S}}{b_{m}\left(2+\left|R^{(m)}\right|\right)}\right) \leq \bar{v}_{i} \quad(\forall s \in S, \forall i \in I)
$$

Based on equation (19), the capacity of each supplier should be enough to provide the required flows of all paths originating from that supplier without using emergency stock. Extra capacity can be reserved in some of the suppliers to compensate for the lack of capacity in other disrupted facilities. By only considering "normal 
conditions" and ignoring other scenarios, equation (19) should be revised as follows in order to exclude this mitigation strategy:

$$
\begin{array}{ll}
\sum_{t=1}^{\left|T^{(i)}\right|} x_{t}^{S}\left(\frac{a_{m \mid m \in t}-\sum_{t=1}^{\left|T^{(m)}\right|} c_{t} x_{t}^{S}}{b_{m}\left(2+\left|R^{(m)}\right|\right)}\right) \leq \bar{v}_{i} & (\forall i \in I) \\
\sum_{t=1}^{\left|T^{(i)}\right|} x_{t}^{S}\left(1-y_{t}^{S}\right)\left(\frac{a_{m \mid m \in t}-\sum_{t=1}^{\left|T^{(m)}\right|} c_{t} x_{t}^{S}}{b_{m}\left(2+\left|R^{(m)}\right|\right)}\right) \leq \sum_{t=1}^{\left|T^{(i)}\right|} x_{t}^{S}\left(\frac{a_{m \mid m \in t}-\sum_{t=1}^{\left|T^{(m)}\right|} c_{t} x_{t}^{S}}{b_{m}\left(2+\left|R^{(m)}\right|\right)}\right) \quad(\forall s \in S-\{n\})
\end{array}
$$

By adding "Extra Reserved Capacity" mitigation strategy, more capacity will be reserved in selected facilities and its corresponding cost will be increased in the objective function (second term in Equation (1) and third term in Equation (22) that will be explained later).

In order to make the design of the SC network resilient to disruptions, the abovementioned model adjustments should be added to the non-robust and competitive SCND model (13-15). The mathematical model of the robust and competitive SCND problem will be as follows:

$$
\begin{aligned}
& \operatorname{Max} \quad \sum_{S=1}^{|S|}\left\{\sum _ { m = 1 } ^ { | M | } \left[\sum_{t=1}^{\left|T^{(m)}\right|}\left(\frac{a_{m \mid m \in t}+\sum_{r=1}^{\left|R^{(m)}\right|} c_{r}+\sum_{t=1}^{\left|T^{(m)}\right|} c_{t} x_{t}^{S}}{\left(2+\left|R^{(m)}\right|\right)}-c_{t}\right)\left(\frac{a_{m}-\sum_{t=1}^{\left|T^{(m)}\right|} c_{t} x_{t}^{S}}{b_{m}\left(2+\left|R^{(m)}\right|\right)}\right) x_{t}^{S}-h_{m}\left(z_{t}-\right.\right.\right. \\
& \left.\left.\left.\left(\frac{a_{m}-\sum_{t=1}^{\left|T^{(m)}\right|} c_{t} x_{t}^{S}}{b_{m}\left(2+\left|R^{(m)}\right|\right)}\right) x_{t}^{S} y_{t}^{S}\right)\right]\right\} p r_{S}-\sum_{i=1}^{|I|}\left(k_{i} \bar{v}_{i}\right)-\sum_{m=1}^{|M|}\left(B_{m} w_{m}\right)-\sum_{i=1}^{|I|}\left(F_{i} v_{i}\right)
\end{aligned}
$$

\section{Subject to:}

$$
\begin{array}{ll}
z_{t} \geq\left(\frac{a_{m \mid m \in t}-\sum_{t=1}^{\left|T^{(m)}\right|} c_{t} x_{t}^{S}}{b_{m}\left(2+\left|R^{(m)}\right|\right)}\right) x_{t}^{S} y_{t}^{S} & (\forall t \in T, \forall s \in S) \\
\sum_{t=1}^{\left|T^{(i)}\right|} x_{t}^{S}\left(1-y_{t}^{S}\right)\left(\frac{a_{m \mid m \in t}-\sum_{t=1}^{\left|T^{(m)}\right|} c_{t} x_{t}^{S}}{b_{m}\left(2+\left|R^{(m)}\right|\right)}\right) \leq \bar{v}_{i} & (\forall s \in S, \forall i \in I) \\
x_{t}^{S} \leq e_{t}^{S}+y_{t}^{S} & \\
y_{t}^{S} \leq 1-e_{t}^{S} & (\forall t \in T, \forall s \in S) \\
\sum_{t=1}^{\left|T^{(m)}\right|} x_{t}^{S}=w_{m} & (\forall t \in T, \forall s \in S) \\
\sum_{s=1}^{|S|} x_{t}^{S} \leq v_{i} & (\forall m \in M, \forall s \in S) \\
\sum_{S=1}^{|S|} x_{t}^{S} \leq w_{m} & \left(\forall t \in T^{(i)}, \forall i \in I\right) \\
0 \leq x_{t}^{S} \leq 1 & \left(\forall t \in T^{(m)}, \forall m \in M\right) \\
v_{i}, w_{m}, y_{t}^{S} \in\{0,1\} & (\forall t \in T, \forall s \in S) \\
z_{t}, \bar{v}_{i} \geq 0 & (\forall m \in M, \forall i \in I, \forall s \in S, \forall t \in T) \\
\hline
\end{array}
$$

Similar to the previous non-resilient case, the first term of the objective function (22) calculates the expected value of the SC's achievable profit over different scenarios. The second term in (22) captures the average inventory-related cost of unused emergency stock. The other terms in the objective function are similar to those in the earlier non-resilient case.

\subsection{SOLUTION APPROACH}

The proposed models in the previous section are mixed integer nonlinear (MIN) mathematical models. The 
main reason for this is the nonlinearity of the equilibrium flow of paths, $\left(\frac{a_{m \mid m \in t}-\sum_{t=1}^{\left|T^{(m)}\right|} c_{t} x_{t}^{n}}{b_{m}\left(2+\left|R^{(m)}\right|\right)}\right) x_{t}^{S}(\forall t \in T, \forall s \in$ $S$ ). The equilibrium price is a linear term but when it is multiplied by the equilibrium flow in the objective function the objective function becomes strictly non-linear. The appearance of equilibrium flow in constraints (23) and (24) makes those constraints non-linear as well.

Based on numerical analysis and as seen in Figure 3, depicting the profit function, first term in objective function (22), is either slightly concave or slightly convex. This specification of profit function derives us to substitute it with a piecewise linear function. There are two main strategies to solve real-scale MIN models: 1) solving exact model with approximating solution method; and 2) solving approximate model with exact solution method. Slight convexity or concavity of the profit function and high accuracy of its piecewise linear approximation, justifies the selection of the second strategy to solve these MIN models.

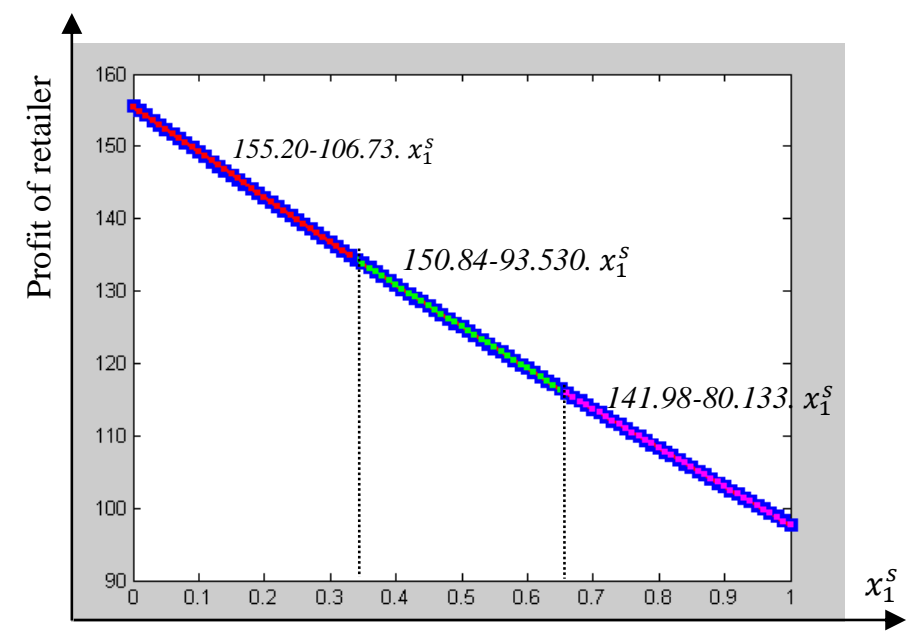

Figure 3. A sample profit function and its three-part linear substitution.

If we break the feasible range of $x_{t}^{S},[0,1]$, into a number of sections - say $K_{t}^{S}$ - and approximate each section with a linear function, the approximate profit function would correspond to (for more detail see Appendix B):

$$
\begin{aligned}
& \left(\frac{a_{m \mid m \in t}+\sum_{r=1}^{\left|R^{(m)}\right|} c_{r}+\sum_{t=1}^{\left|T^{(m)}\right|} c_{t} x_{t}^{n}}{\left(2+\left|R^{(m)}\right|\right)}-c_{t}\right)\left(\frac{a_{m}-\sum_{t=1}^{\left|T^{(m)}\right|} c_{t} x_{t}^{n}}{b_{m}\left(2+\left|R^{(m)}\right|\right)}\right) x_{t}^{S}= \\
& \left\{\begin{array}{ccc}
\theta_{t, 1}^{S}+\vartheta_{t, 1}^{S} x_{t}^{S} & \text { if } & 0 \leq x_{t}^{S}<\delta_{t, 1}^{s} \\
\theta_{t, 2}^{S}+\vartheta_{t, 2}^{S} x_{t}^{S} & \text { if } & \delta_{t, 1}^{s} \leq x_{t}^{S}<\delta_{t, 2}^{s} \\
\cdots & & \cdots \\
\theta_{t, K_{t}^{s}}^{s}+\vartheta_{t, K_{t}^{s}}^{s} x_{t}^{S} & \text { if } & \delta_{t, K_{t}^{s}-1}^{s} \leq x_{t}^{S}<1
\end{array} \quad(\forall t \in T, \forall s \in S)\right.
\end{aligned}
$$

A similar approach is used to linearize the assigned equilibrium flow of the paths. Our numerical analysis shows the similar slight convexity and concavity in the equilibrium flows of the paths (see Figure 4). See Appendix C for more details. 


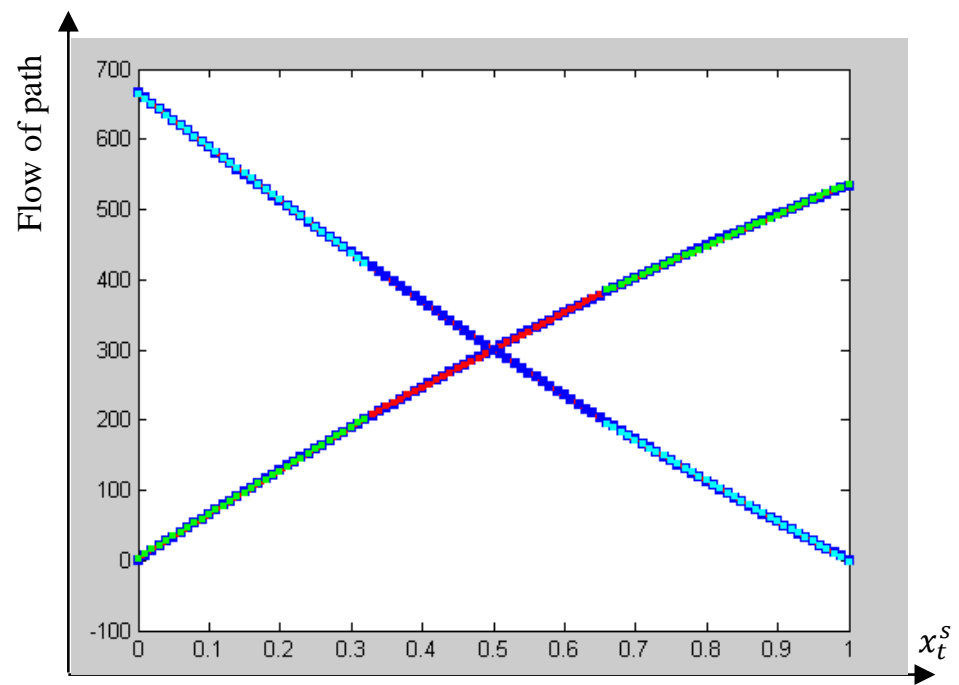

Figure 4. Two sample path flows and their three-part linear substitutions.

(Dark blue and light blue line indicates flow of first sample path. Green and red line indicate flow of second sample path)

\section{EXPERIMENTAL RESULTS}

In this section, the various scenarios discussed earlier are analyzed using the data from the case company in order to extract the related insights.

\subsection{Case study analysis: SMAC company}

The potential network structure of the case study and its usable paths are shown in Figure 5. The cost of producing and selling through each path and the cost of product supplied by rival are summarized in Table 1. The annual fixed costs of locating a retailer in all the existing markets are the same and equal to 10 . The cost of reserving one unit of capacity at any of the suppliers is 0.01 . The inventory-related cost for each unit of product is 0.01 .

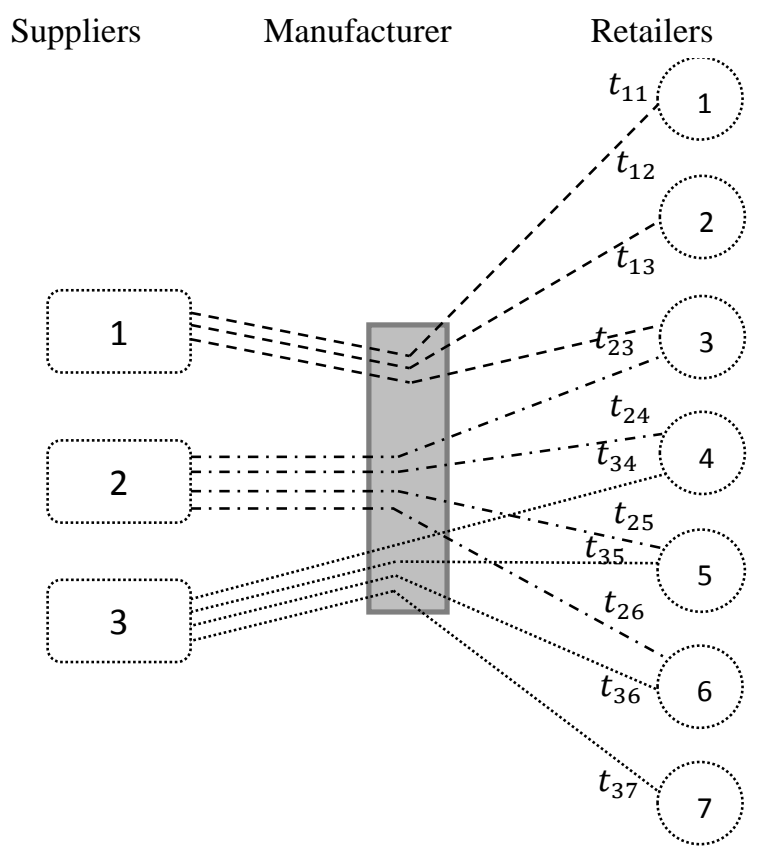

Figure 5. Potential network structure of SMAC for the manufacture of reverse idler gear shafts. 
Table 1. Cost of producing unit product through potential paths.

\begin{tabular}{|c|c|c|c|c|c|c|c|}
\hline \multicolumn{2}{|c|}{ Retailer 1 } & \multicolumn{2}{c|}{ Retailer 2 } & \multicolumn{2}{c|}{ Retailer 3 } & \multicolumn{2}{|c|}{ Retailer 4 } \\
\hline$t_{11}$ & 1.85 & $t_{12}$ & 1.80 & $t_{13}$ & 1.80 & $t_{24}$ & 1.65 \\
\hdashline Rival & 1.77 & Rival & 1.77 & $t_{23}$ & 1.65 & $t_{34}$ & 1.50 \\
\hdashline- & - & - & & Rival & 1.70 & Rival & 1.70 \\
\hline Retailer 5 & \multicolumn{2}{|c|}{ Retailer 6 } & Retailer 7 & & \\
\hline$t_{25}$ & 1.60 & $t_{26}$ & 1.60 & $t_{37}$ & 1.50 & & \\
\hdashline$t_{35}$ & 1.50 & $t_{36}$ & 1.50 & Rival & 1.55 & & \\
\hline Rival & 1.70 & Rival & 1.70 & - & - & & \\
\hline
\end{tabular}

It is assumed that the first supplier is more costly than the others but $100 \%$ reliable. Supplier 2 is the next most expensive, but its production facilities might be out-of-order with 0.1 probability based on the historic data. The third supplier is the cheapest, but has the highest probability of disruption, which is 0.25 . Thus four scenarios can be defined for this problem. First scenario represents normal conditions when all suppliers are available. The probability of the normal scenario is $1-(0.075+0.225+0.025)=0.675$. In the second and third scenarios, with the probability of 0.075 and 0.225 respectively, the second and third suppliers are disrupted. The fourth scenario considers disruptions in both second and third suppliers, and its probability of occurrence is $0.25 \times 0.1=0.025$. In this setting, we assume that there is no probability of disruption in the connecting links of the SC. Incorporating such considerations into the model would only augment the number of scenarios. Regarding the price function of products in the markets, we assume that $a_{m}=2$ and $b_{m}=2.5 \times 10^{-4}$ for all markets.

The proposed models in the paper are mixed integer nonlinear (MIN) mathematical models with binary variables. The complexity and solution time of the models depends on the number of binary variables. In the models, there are $|I|+|M|+|S| .|T|$ binary variables. In the case study problem, there are three potential suppliers, $|I|=3$, one manufacturer, and seven potential markets, $|M|=7$, with eleven potential paths, $|T|=11$. Also disruptions in the chain are modelled as four scenarios. Therefore, there are 54 binary variables in the models and solution time is a couple of seconds. We solve this model using CPLEX Concert Technology on a Dell laptop computer with Windows 10, Intel i7 processor, and 8 GB of installed RAM. Assuming that facilities (suppliers and markets) and disruption scenarios increase in the same proportions for higher number of manufacturers, by increasing the number of manufacturers up to $9\left(\leq \frac{500}{|I|+|M|+|S| \cdot|T|=45}\right)$ the number of binary variables in the models stays less than 500. According to our experience, such MIN models with less than 500 binary variables are easily solvable in a reasonable computational time (Rezapour et al., 2015).

\subsection{Implications and insights}

\section{Implications of Creating Resilience for Supply Capability}

The first and second Research Questions (RQ1 and RQ2) that are going to be answered in this section are as follows:

- RQ1: What is the effect of ignoring disruptions on the supply capability of the SC?

- RQ2: How different risk mitigation strategies contribute to improving the supply capability of the SC?

To answer these questions, first we solve the non-resilient model by ignoring the possible disruptions (first test problem). Obtaining these results helps us to evaluate the benefits of taking into account the possibility of disruptions in the model. The results of solving this model are visualized in Figure 6. Model does not find it profitable enough for the $\mathrm{SC}$ to establish a retailer to service the first market. 


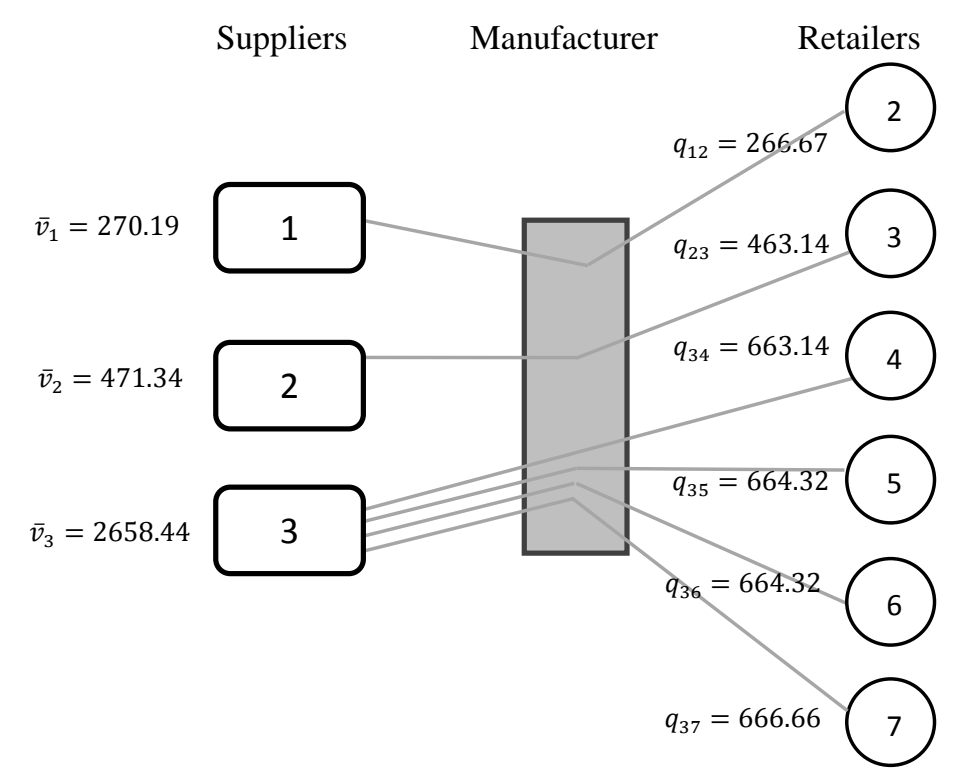

Figure 6. Network structure of SMAC in the first test problem.

In this case, the profit of the SC in normal conditions is 665.26. However, when disruptions occur to the second, to the third or to both suppliers then $\left\{t_{23}\right\},\left\{t_{34}, t_{35}, t_{36}, t_{37}\right\}$ and $\left\{t_{23}, t_{34}, t_{35}, t_{36}, t_{37}\right\}$ path sets become inoperative. As a result its supply capability reduces by $14 \%, 78 \%$ and $92 \%$ respectively. These percentages are calculated based on the difference in supply quantity to the markets in the normal scenario compared with the other scenarios. This decreases the profit to 481.173, 76.98 and 15.11 respectively. This means that the SC's long-term expected profit would reduce to 502.83 , leaving aside the effects that poor service levels in the competitive markets would have on the reputation of the SC.

In the next step, we solve the resilient model of the problem (second test problem). This model is a fourscenario model incorporating all disruption possibilities concurrently and maximizes the average profit of the supply chains. Then once we have the optimal solution of this model, we can evaluate and find profit, price, market share, etc. under each scenario.

In this problem, however, the disruption probability is incorporated into the model; the SC can use multiple-sourcing, reserving extra capacity and keeping emergency inventory at the retailers to mitigate the effects of disruptions and ensure a sustained supply of products to the markets. The results are visualized in Figure 7. As observed, in this case there is some extra capacity at the first supplier and there is some extra emergency stock, $z_{t}$, at the fourth, fifth, sixth and seventh retailers which are not used under normal conditions (Scenario 1) but, as will be explained later, are kept to be used in the event of disruption. 


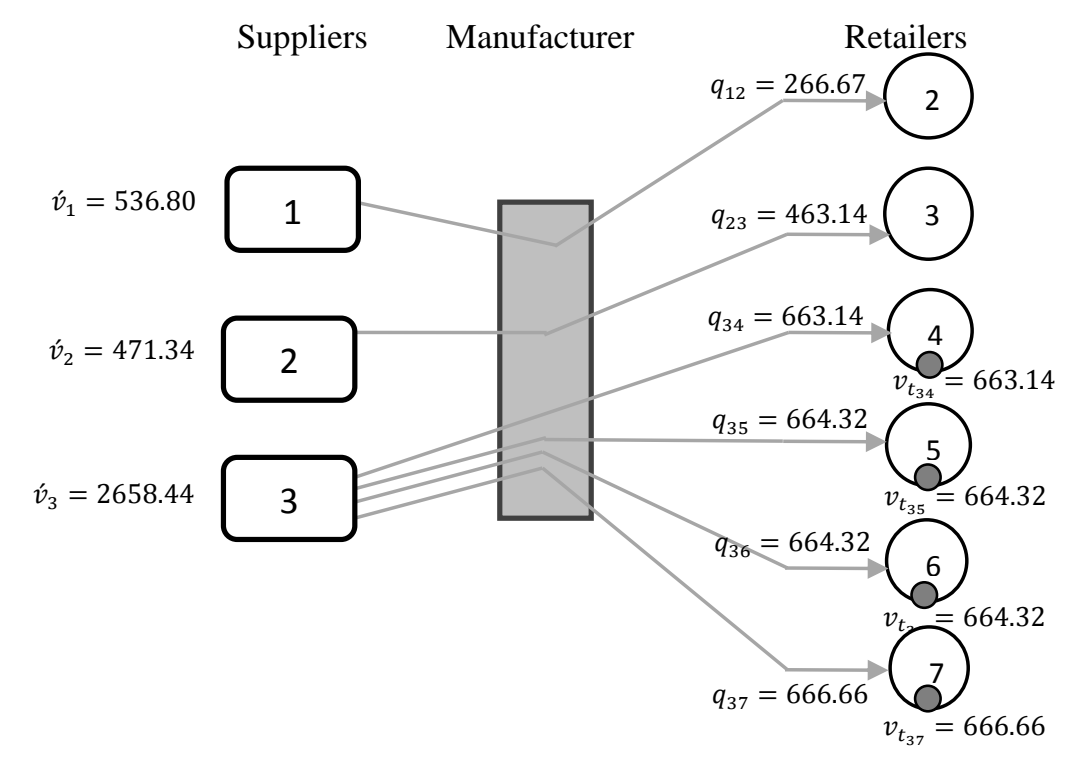

Figure 7. Network structure of SMAC in the second test problem (Scenario 1).

It should be noted that $q_{i j}$ is the quantity of product flow through the path originating from supplier $i$ and ending at retailer $j$. Figure 8 represents the product flow in the network of the resilient SC in the second scenario in which supplier 2 is disrupted. In this case, supplier 1 is substituted for supplier 2 and its extra capacity is used on path $t_{13}$ to supply products to market 3. Due to "extra capacity" mitigation strategy, the supply capability of the SC only reduces $5.6 \%$ in comparison to the normal condition. The supply capability of the resilient SC in scenario 2 is $8.4 \%$ higher than the non-resilient SC's.

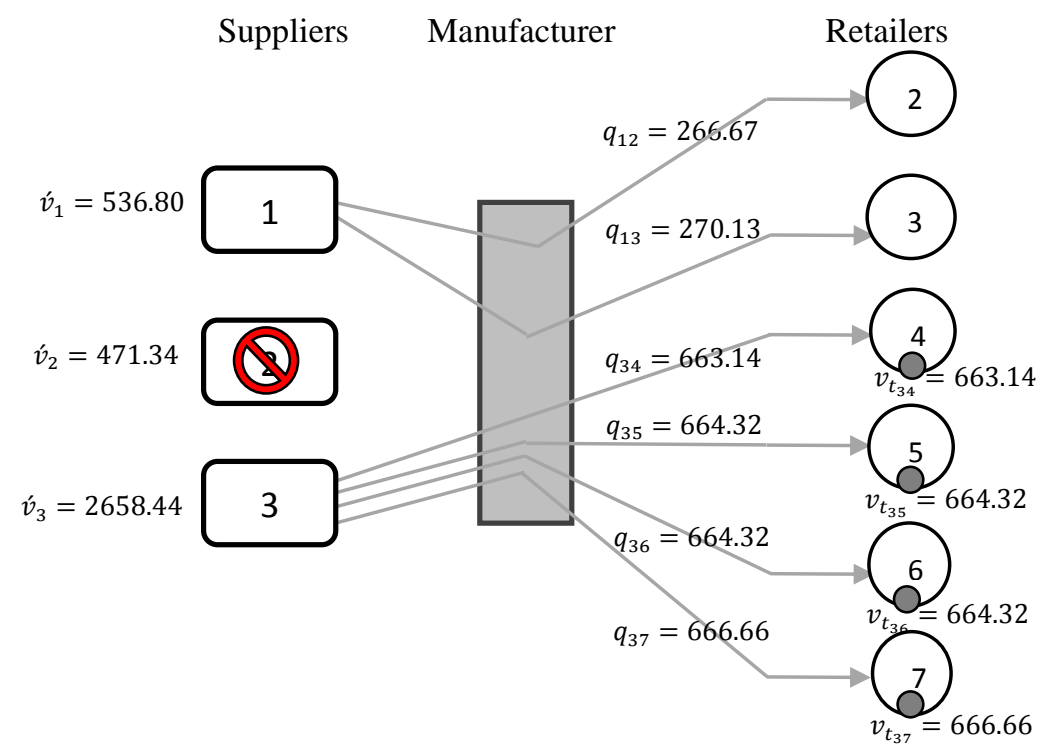

Figure 8. Product flow in the resilient SC in the second scenario.

Figure 9 represents the product flow in the network of the resilient SC in the third scenario in which supplier 3 is inoperative. In this case, all the supply paths originating from the third supplier, serving the markets 4, 5, 6 and 7, are unavailable. So the SC uses the extra inventory kept at the retailers to compensate for the disrupted supplier. Due to emergency stock mitigation strategy, the supply capability of SC does not change in the third scenario in comparison to the normal conditions (scenario 1). The supply capability of the resilient $\mathrm{SC}$ in scenario 3 is $78 \%$ higher than that of the non-resilient SC. 


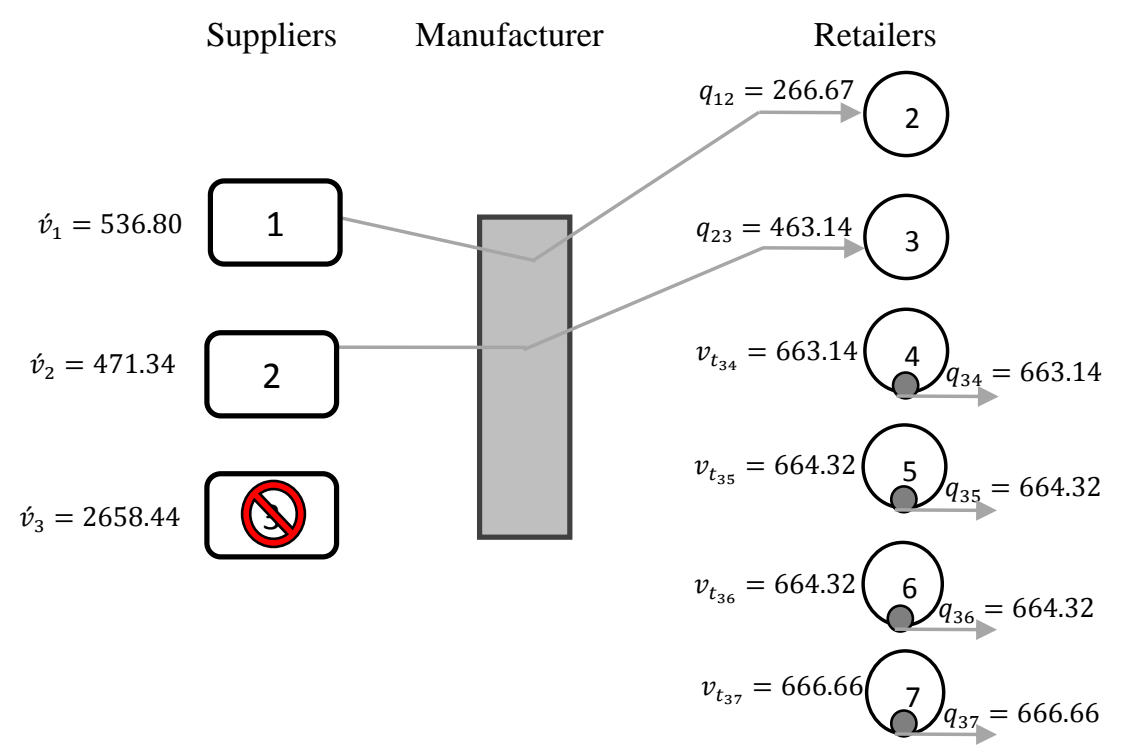

Figure 9. Product flow in the resilient SC in the third scenario.

Figure 10 illustrates the product flow in the network of the resilient SC in scenario 4 in which both suppliers 2 and 3 are inoperative. In this case, the extra capacity of supplier 1 and the inventories at retailers are used to fulfil the demands of markets 3, 4, 5, 6, and 7. Supply quantities to the markets in Figure 10 show that the supply capability of the resilient SC in scenario 4 is $86 \%$ higher than that of the non-resilient SC. The share of the "Emergency stock" and "Extra Capacity" in this supply capability improvement is $90.8 \%$ and 9.2\% respectively.

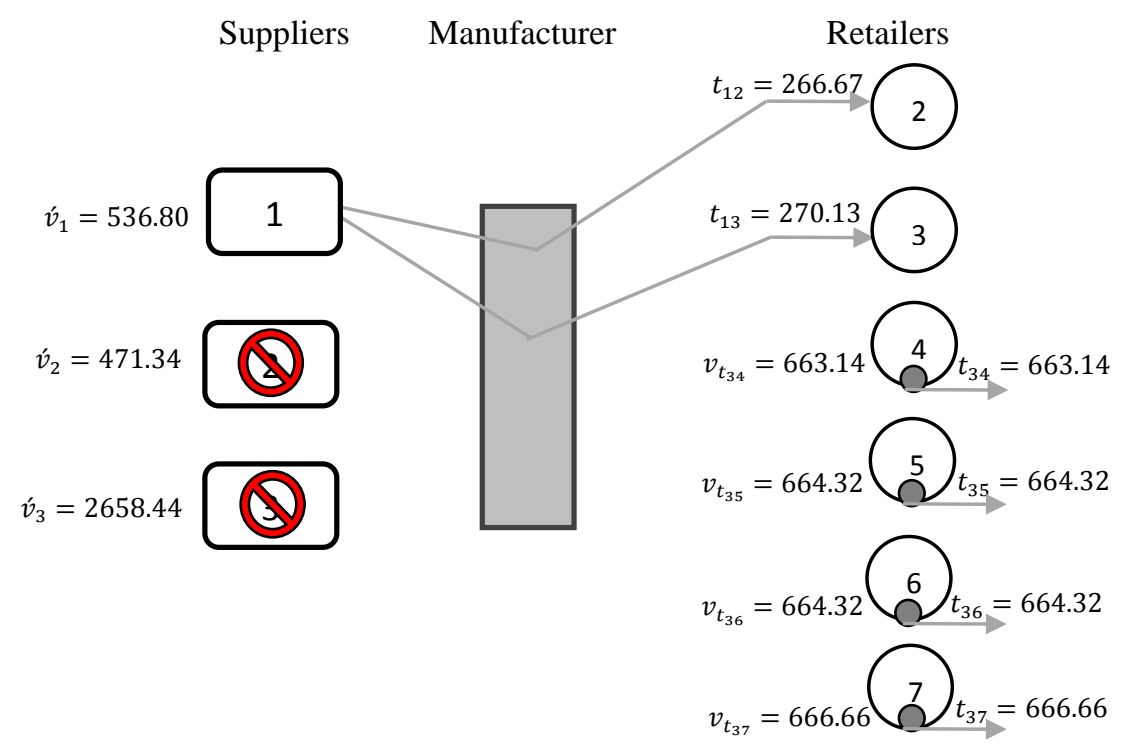

Figure 10. Product flow in the resilient SC in the fourth scenario.

By considering the probability of the scenarios, we conclude that:

The average supply capability of the resilient SC is $\sim 20.34 \%$ higher than the non-resilient SC. The average share of "Emergency stock" and "Extra Capacity" in this improvement is $\sim 15.255 \%$ and $\sim 5.085 \%$ respectively.

Supply quantity of the SC in the resilient and non-resilient cases and in the event of different disruption scenarios is shown in Table 2. 
Table 2. Comparison of the SC's supply quantity in the resilient and non-resilient cases.

\begin{tabular}{|c|c|c|c|c|c|}
\hline \multirow{5}{*}{$\begin{array}{c}\text { Resilient } \\
\text { SC }\end{array}$} & \multicolumn{2}{|c|}{ Supply } & Mean supply & Standard deviation of supply & The worst case supply \\
\hline & Scenario 1 & 3388.25 & \multirow{4}{*}{3368.95} & \multirow{4}{*}{ - } & \multirow{4}{*}{3195.24} \\
\hline & Scenario 2 & 3195.24 & & & \\
\hline & Scenario 3 & 3388.25 & & & \\
\hline & Scenario 4 & 3195.24 & & & \\
\hline \multirow{4}{*}{$\begin{array}{l}\text { Non- } \\
\text { resilient SC }\end{array}$} & Scenario 1 & 3388.25 & \multirow{4}{*}{2677.32} & \multirow{4}{*}{34.05} & \multirow{4}{*}{266.67} \\
\hline & Scenario 2 & 2925.11 & & & \\
\hline & Scenario 3 & 729.81 & & & \\
\hline & Scenario 4 & 266.67 & & & \\
\hline \multicolumn{3}{|c|}{ Improvement } & $\sim 26 \%$ & $\sim 447 \%$ & 1198\% \\
\hline
\end{tabular}

The results summarized in Table 2 show that equipping the SCs with risk mitigation strategies to make them resilient improves their average supply capability and reduces the standard deviation of supply. This, essentially, means that there is a tradeoff between SCs' average supply capability and the standard deviation of their supply. We can increase the resilience of a SC by adding more risk mitigation strategies. Higher resilience means higher average and lower standard deviation for the SC's supply quantity.

\section{Implications of Creating Resilience for the SC profit}

In this section we answer the third research question (RQ3): What is the effect of ignoring disruptions on the SC profit?

In the resilient case, the profit of the SC in the normal conditions is 638.67 , lower than the profit of the non-resilient model which was 665.263. This difference can be considered as the resilience cost to the SC, given that risk mitigation strategies inevitably impose some costs on the system. However, it should be noted that in the event of disruption to the second, third or both of these suppliers, the profit of the SC could be kept at 576.8, 665.2 and 603.39 respectively. The standard deviation of the SC profit in the resilient model is 4.67 but for the non-resilient model it is 314.51 .

As can be seen in Table 3, the average profit of the resilient SC is $\sim 27 \%$ better than the non-resilient SC. Being resilient reduces the standard deviation of the SC's profit by $\sim 341 \%$ and improves the SC's worst case profit $\sim 38$ fold.

Based on the results summarized in Table 3, we conclude:

The average profit of the resilient SC is $\sim 27 \%$ higher than the non-resilient SC.

Table 3. Comparison of the SC's profit in the resilient and non-resilient cases.

\begin{tabular}{|c|c|c|c|c|c|}
\hline \multirow{5}{*}{$\begin{array}{c}\text { Resilient } \\
\text { model }\end{array}$} & \multicolumn{2}{|c|}{ Profit } & Mean Profit & Standard deviation of profit & The worst case profit \\
\hline & Scenario 1 & 638.67 & \multirow{4}{*}{639.11} & \multirow{4}{*}{4.67} & \multirow{4}{*}{576.80} \\
\hline & Scenario 2 & 576.80 & & & \\
\hline & Scenario 3 & 665.26 & & & \\
\hline & Scenario 4 & 603.39 & & & \\
\hline \multirow{4}{*}{$\begin{array}{c}\text { Non- } \\
\text { resilient } \\
\text { model }\end{array}$} & Scenario 1 & 665.26 & \multirow{4}{*}{502.83} & \multirow{4}{*}{15.94} & \multirow{4}{*}{15.11} \\
\hline & Scenario 2 & 481.17 & & & \\
\hline & Scenario 3 & 76.98 & & & \\
\hline & Scenario 4 & 15.11 & & & \\
\hline \multicolumn{3}{|c|}{ Improvement } & $\sim 27 \%$ & $\sim 341 \%$ & $\sim 3817 \%$ \\
\hline
\end{tabular}


Incorporating risk mitigation strategies improves the supply capability of SCs in the event of disruption. Higher supply to markets results in an enhanced service to customers that leads to higher income. However, incorporating risk mitigation strategies comes at a cost. Therefore, a tradeoff should be established between the cost of creating resiliency and its revenue. The model developed in this paper helps us to find the best tradeoff between the cost and revenue of resilience in a SC in a way to maximize its profit. In the case problem, the most profitable compromise happens when enough risk mitigation strategies are added to the SC and the average supply reduction in disruptions decreases to $26 \%$.

\section{Implications of Creating Resilience for the Market Prices and Shares}

In this section, we compare the market share and the retail price in different markets in the resilient and nonresilient models. The results are summarized in Table 4 and displayed in Figures 11 and 12. The values presented in this table corresponding to retail price and market share are calculated as follows:

- Retail price in the resilient case: to compute the retail price in each market and in each scenario, first we solve the model formulated in 22-32 for the case problem and find optimal values for $x_{t}^{S}(\forall s \in S, \forall m \in$ $\left.M, \forall t \in T^{(m)}\right)$. By substituting these variables in $\frac{a_{m}+\sum_{r=1}^{\left|R^{(m)}\right|} c_{r}+\sum_{t=1}^{\left|T^{(m)}\right|} c_{t} x_{t}^{S}}{\left(2+\left|R^{(m)}\right|\right)}(\forall m \in M$ and $\forall s \in S)$, we compute equilibrium retail price in each market and in each scenario.

- Market share of SMAC in the resilient case: to compute the market share of SMAC in each market and in each scenario, first we solve the model formulated in 22-32 for the case problem and find optimal values for $x_{t}^{S} \quad\left(\forall s \in S, \forall m \in M, \forall t \in T^{(m)}\right)$. By substituting these variables in $\sum_{t=1}^{\left|T^{(m)}\right|}\left(\frac{a_{m}-\sum_{t=1}^{\left|T^{(m)}\right|} c_{t} x_{t}^{s}}{b_{m}\left(2+\left|R^{(m)}\right|\right)}\right) \cdot x_{t}^{S}$, the supply quantity of SMAC to market $m(\forall m \in M)$ in scenario $s(\forall s \in$ $S)$ is computed. Equation $\left(\frac{a_{m}-c_{r}}{b_{m}\left(2+\left|R^{(m)}\right|\right)}\right)\left(\forall r \in R^{(m)}\right.$ and $\left.\forall m \in M\right)$ determines the supply quantity of rival $r\left(\forall r \in R^{(m)}\right.$ and $\left.\forall m \in M\right)$ to market $m$. Therefore, the market share of SMAC in market $m$ $(\forall m \in M)$ and in scenario $s(\forall s \in S)$ is computed as follows:

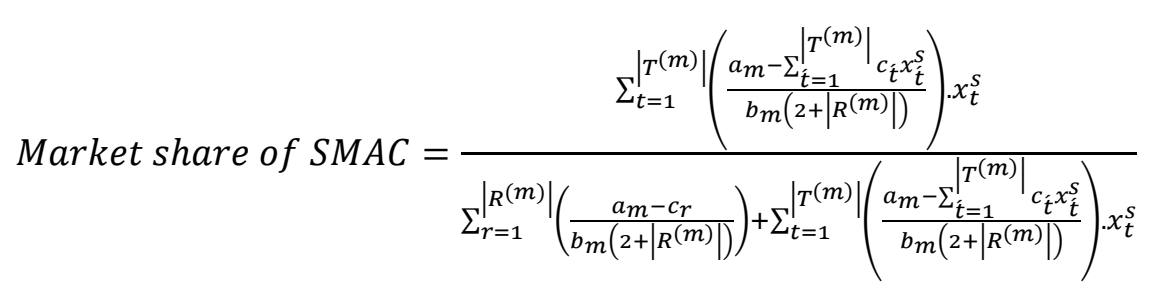

The market share of rival $r\left(\forall r \in R^{(m)}\right)$ in market $m(\forall m \in M)$ and in scenario $s(\forall s \in S)$ is computed as follows:

$$
\text { Market share of rival } r \text { in market } m=\frac{\left(\frac{a_{m}-c_{r}}{b_{m}\left(2+\left|R^{(m)}\right|\right)}\right)}{\sum_{r=1}^{R^{(m)} \mid}\left(\frac{a_{m}-c_{r}}{b_{m}\left(2+\left|R^{(m)}\right|\right)}\right)+\sum_{t=1}^{T^{(m)} \mid}\left(\frac{a_{m}-\sum_{t=1}^{T^{(m)} \mid} c_{t} x_{t}^{s}}{b_{m}\left(2+\left|R^{(m)}\right|\right)}\right) \cdot x_{t}^{s}}
$$

- Market share of SMAC in the non-resilient case: to compute market share of SMAC in each market and 
in each scenario, first we solve the model formulated in 13-15 and find optimal values for $x_{t}^{n}(\forall m \in$ $\left.M, \forall t \in T^{(m)}\right)$. By substituting these variables in $\sum_{t=1}^{\left|T^{(m)}\right|}\left(\frac{a_{m}-\sum_{t=1}^{T^{(m)} \mid} c_{t} x_{t}^{n}}{b_{m}\left(2+\left|R^{(m)}\right|\right)}\right) \cdot x_{t}^{n} \cdot\left(1-e_{t}^{S}\right)$, the supply quantity of SMAC to market $m(\forall m \in M)$ in scenario $s(\forall s \in S), q_{m}^{s}$, is computed. Then the market shares of SMAC and its competitors can be calculated following a logic similar to the non-resilient case.

- Retail price in the non-resilient case: after computing $q_{m}^{s}$ values, the retail price in market $m(\forall m \in M)$ and in scenario $s(\forall s \in S), p_{m}^{S}$, is computed as follows:

$$
p_{m}^{S}=a_{m}-b_{m}\left(q_{m}^{S}+\sum_{r=1}^{\left|R^{(m)}\right|}\left(\frac{a_{m}-c_{r}}{b_{m}\left(2+\left|R^{(m)}\right|\right)}\right)\right)
$$

As can be seen, a resilient SC is always able to capture a larger market share than a non-resilient one. Apparently, the mitigation strategies help the SC to stabilize its supply quantities. This not only leads to higher profit but also in the long run positively affects the reputation of the company and improves competitiveness.

Table 4. Market share and retail prices in the markets.

\begin{tabular}{|c|c|c|c|c|c|c|c|c|c|c|c|}
\hline \multirow[b]{2}{*}{$\frac{\bar{d}}{\substack{i \\
\Sigma}}$} & \multirow[b]{2}{*}{ 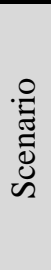 } & \multicolumn{5}{|c|}{ Resilient } & \multicolumn{5}{|c|}{ Non-resilient } \\
\hline & & Price & $\begin{array}{c}\text { Supply } \\
\text { quantity by } \\
\text { SMAC }\end{array}$ & 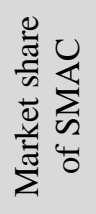 & $\begin{array}{c}\text { Supply } \\
\text { quantity by the } \\
\text { rival }\end{array}$ & 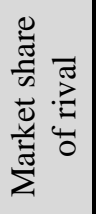 & Price & $\begin{array}{c}\text { Supply } \\
\text { quantity by } \\
\text { SMAC }\end{array}$ & 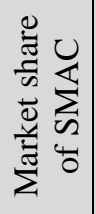 & $\begin{array}{c}\text { Supply quantity } \\
\text { by the rival }\end{array}$ & 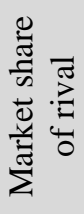 \\
\hline \multirow{4}{*}{2} & 1 & 1.850 & 266.66 & .47 & 306.66 & .53 & 1.850 & 266.66 & .47 & 306.66 & .53 \\
\hline & 2 & 1.850 & 266.66 & .47 & 306.66 & .53 & 1.850 & 266.66 & .47 & 306.66 & .53 \\
\hline & 3 & 1.850 & 266.66 & .47 & 306.66 & .53 & 1.850 & 266.66 & .47 & 306.66 & .53 \\
\hline & 4 & 1.850 & 266.66 & .47 & 306.66 & .53 & 1.850 & 266.66 & .47 & 306.66 & .53 \\
\hline \multicolumn{2}{|c|}{ Avg. } & 1.850 & 266.66 & .47 & 306.66 & .53 & 1.850 & 266.66 & .47 & 306.66 & .53 \\
\hline \multirow{4}{*}{3} & 1 & 1.783 & 466.67 & .53 & 400 & .47 & 1.783 & 466.67 & .53 & 400.00 & .47 \\
\hline & 2 & 1.833 & 266.67 & .40 & 400 & .60 & 1.850 & 0 & .00 & 600.00 & 1.0 \\
\hline & 3 & 1.783 & 466.67 & .53 & 400 & .47 & 1.783 & 466.67 & .53 & 400.00 & .47 \\
\hline & 4 & 1.833 & 266.67 & .40 & 400 & .60 & 1.850 & 0 & .00 & 600.00 & 1.0 \\
\hline \multicolumn{2}{|c|}{ Avg. } & 1.789 & 441.67 & .53 & 400 & .47 & 1.791 & 408.33 & .49 & 425.00 & .51 \\
\hline \multirow{4}{*}{4} & 1 & 1.733 & 666.67 & .62 & 400 & .38 & 1.733 & 666.67 & .62 & 400 & .38 \\
\hline & 2 & 1.733 & 666.67 & .62 & 400 & .38 & 1.733 & 666.67 & .62 & 400 & .38 \\
\hline & 3 & 1.733 & 666.67 & .62 & 400 & .38 & 1.85 & 0 & .00 & 600 & 1.0 \\
\hline & 4 & 1.733 & 666.67 & .62 & 400 & .38 & 1.85 & 0 & .00 & 600 & 1.0 \\
\hline \multicolumn{2}{|c|}{ Avg. } & 1.733 & 666.67 & .62 & 400 & .38 & 1.765 & 483.33 & .45 & 455 & .55 \\
\hline \multirow{4}{*}{5} & 1 & 1.733 & 666.67 & .62 & 400 & .38 & 1.733 & 666.67 & .62 & 400 & .38 \\
\hline & 2 & 1.733 & 666.67 & .62 & 400 & .38 & 1.733 & 666.67 & .62 & 400 & .38 \\
\hline & 3 & 1.733 & 666.67 & .62 & 400 & .38 & 1.850 & 0 & .00 & 600 & 1.0 \\
\hline & 4 & 1.733 & 666.67 & .62 & 400 & .38 & 1.850 & 0 & .00 & 600 & 1.0 \\
\hline \multicolumn{2}{|c|}{ Avg. } & 1.733 & 666.67 & .62 & 400 & .38 & 1.765 & 483.33 & .45 & 455 & .55 \\
\hline \multirow{4}{*}{6} & 1 & 1.733 & 666.67 & .62 & 400 & .38 & 1.733 & 666.67 & .62 & 400 & .38 \\
\hline & 2 & 1.733 & 666.67 & .62 & 400 & .38 & 1.733 & 666.67 & .62 & 400 & .38 \\
\hline & 3 & 1.733 & 666.67 & .62 & 400 & .38 & 1.850 & 0 & .00 & 600 & 1.0 \\
\hline & 4 & 1.733 & 666.67 & .62 & 400 & .38 & 1.850 & 0 & .00 & 600 & 1.0 \\
\hline \multicolumn{2}{|c|}{ Avg. } & 1.733 & 666.67 & .62 & 400 & .38 & 1.765 & 483.33 & .45 & 455 & .55 \\
\hline \multirow{4}{*}{7} & 1 & 1.683 & 666.67 & .53 & 600 & .47 & 1.683 & 666.67 & .53 & 600 & .47 \\
\hline & 2 & 1.683 & 666.67 & .53 & 600 & .47 & 1.683 & 666.67 & .53 & 600 & .47 \\
\hline & 3 & 1.683 & 666.67 & .53 & 600 & .47 & 1.775 & 0 & .00 & 900 & 1.0 \\
\hline & 4 & 1.683 & 666.67 & .53 & 600 & .47 & 1.775 & 0 & .00 & 900 & 1.0 \\
\hline \multicolumn{2}{|c|}{ Avg. } & 1.683 & 666.67 & .53 & 600 & .47 & 1.708 & 483.33 & .38 & 537.5 & .62 \\
\hline
\end{tabular}


It is interesting to note that a steady supply of a product by the resilient SC stabilizes the retail price of that product in the market. As seen in Table 2, fluctuations in the retail price of the product are much greater in the non-resilient SC than in the resilient one. Analyzing the results of Table 3 answers the questions regarding the effect of ignoring disruptions on the market shares and price as follows:

The market share stability of the resilient $S C$ is $\sim 33.5$ percent higher than the stability of the non-resilient $S C$ without mitigation strategies.

The price stability of the resilient SC is $\sim 6.7$ percent higher than that of the SC without mitigation strategies.

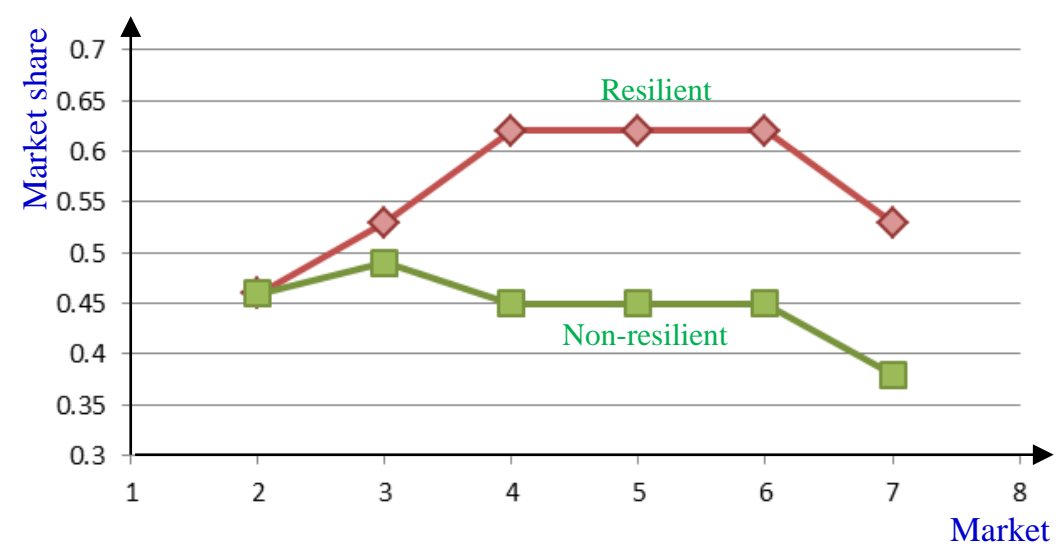

Figure 11. Market shares of resilient and non-resilient $\mathrm{SCs}^{3}$.

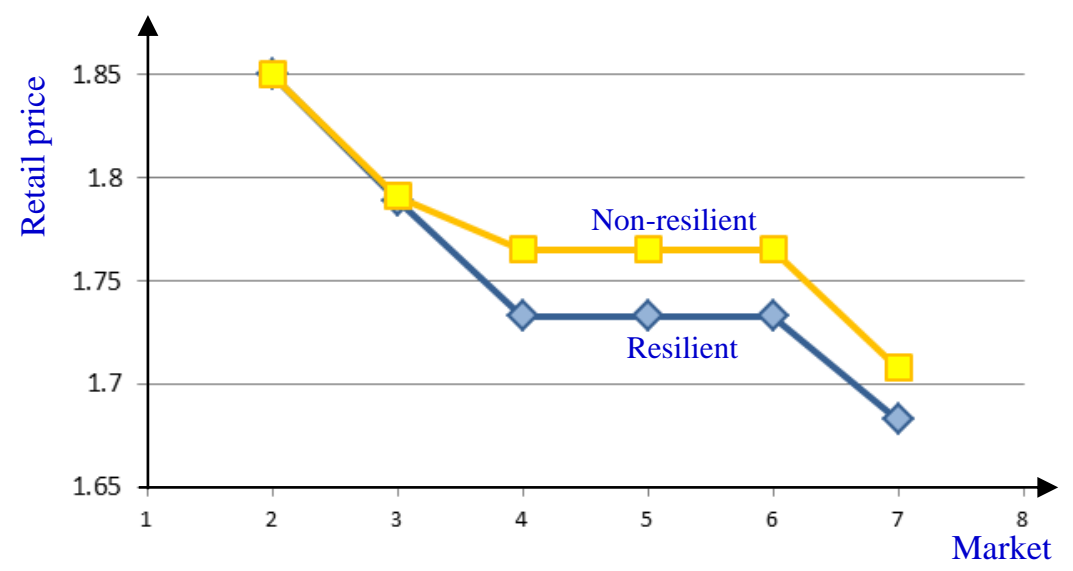

Figure 12. Retail prices of resilient and non-resilient SCs.

\section{Contribution of Risk Mitigation Strategies to the SC Profit}

In order to gain insights on the value of mitigation strategies, we develop another model in which no emergency stock is held. For this, variables $y_{t}^{s}$ and $z_{t}$ are removed from the problem formulation and the term relating to holding cost is also deleted from the objective function. In this case, it is not required to have (23) to

\footnotetext{
${ }^{3}$ Only one potential path ends at Market 7 represented by $t_{37}$. This path originates from the third supplier. So in the third and fourth scenarios when the third supplier is out-of-use, the SC completely losses its market share in Market 7. Therefore, as seen in Figure 11, the SC's average market share is low in Market 7 in the non-resilient case. Moreover, the cost of producing and distributing product unit by Market 7's rival (\$1.55) is very low in comparison with other markets' rivals (for example it is $\$ 1.7$ for Markets 4, 5, and 6). Therefore, as seen in Figure 12, the equilibrium retail price is lower in Market 7 in comparison with other markets.
} 
take care of the level of emergency stock at the retailer or (26) to allow the usage of emergency stock once disruption occurs. Also $y_{t}^{s}$ should be set equal to 0 in (24). The results related to profit of the results from solving this simpler model are summarized in Table 5.

Table 5. Comparison of resilient models with and without emergency stock.

\begin{tabular}{|c|c|c|c|c|c|}
\hline Strategy & \multicolumn{2}{|c|}{ Profit } & Mean profit & $\begin{array}{c}\text { Standard deviation } \\
\text { of profit }\end{array}$ & $\begin{array}{c}\text { Worst case } \\
\text { profit }\end{array}$ \\
\hline \multirow{4}{*}{$\begin{array}{l}\text { Resilient model } \\
\text { with emergency } \\
\text { stock }\end{array}$} & Scenario 1 & 638.67 & \multirow{4}{*}{639.11} & \multirow{4}{*}{4.67} & \multirow{4}{*}{576.80} \\
\hline & Scenario 2 & 576.80 & & & \\
\hline & Scenario 3 & 665.26 & & & \\
\hline & Scenario 4 & 603.39 & & & \\
\hline \multirow{4}{*}{$\begin{array}{l}\text { Resilient model } \\
\text { without } \\
\text { emergency stock }\end{array}$} & Scenario 1 & 665.2630 & \multirow{4}{*}{569.86} & \multirow{4}{*}{12.75} & \multirow{4}{*}{15.11} \\
\hline & Scenario 2 & 603.3930 & & & \\
\hline & Scenario 3 & 334.1341 & & & \\
\hline & Scenario 4 & 15.11000 & & & \\
\hline
\end{tabular}

As seen in this table, removing the extra inventory strategy improves the profitability of the SC in scenario 1. It is an intuitive finding since the cost of holding inventory is removed from the profit function. However, in the other scenarios, such as 3 and 4 in which a more economical supplier is unavailable, the profit decreases significantly. Lack of extra inventory and the unavailability of two suppliers in scenario 4 limit the supply capability of the SC. Consequently, the expected profit of the simple model decreases and the standard deviation of profit in different scenarios increases significantly. As shown in the final column of Table 5, the worst-case profit in this simpler version of the resilient model is markedly lower than the worst-case profit of the original resilient model which contained all contingency strategies. This result highlights the importance of the emergency stock as a powerful risk mitigation strategy. The profits in Tables 3 and 5 are compared to determine the contribution of risk mitigation strategies in improving the profitability of the resilient SC as follows:

The average profit of the resilient SC is $\sim 27 \%$ higher than the non-resilient SC. The share of "Emergency Stock" and "Extra Capacity" in this profit improvement is 50\% for both strategies.

Because of the higher share of "Emergency Stock" in the supply capability of the SC, clearly this strategy is more costly than the "Extra Capacity" strategy. The roles of these strategies in reducing profit variation and improving worst-case profit of the case problem are stated as follows:

The share of "Emergency Stock" and "Extra Capacity" in reducing the profit variation is $\sim 72 \%$ and $\sim 28 \%$ respectively.

The share of "Emergency Stock" and "Extra Capacity" in improving the SC worst case profit is $\sim 100 \%$ and $\sim 0 \%$ respectively.

Detailed results showing the performance of the SC in different scenarios are presented in Figures 13-15. Figure 13 represents the flow in the SC in the first scenario when there is no disruption. The only difference between this figure and that showing the resilient model (Figure 3) is in the reserved capacity of the second 
supplier. Intuitively, where there are fewer mitigation policies in place, the results from this model indicate that more capacity should be reserved at the second supplier in order to hedge against disruption and compensate for the unavailability of the third supplier. The network flows of the two models are almost similar. The main difference between the results of these two models is in the third and fourth scenarios.

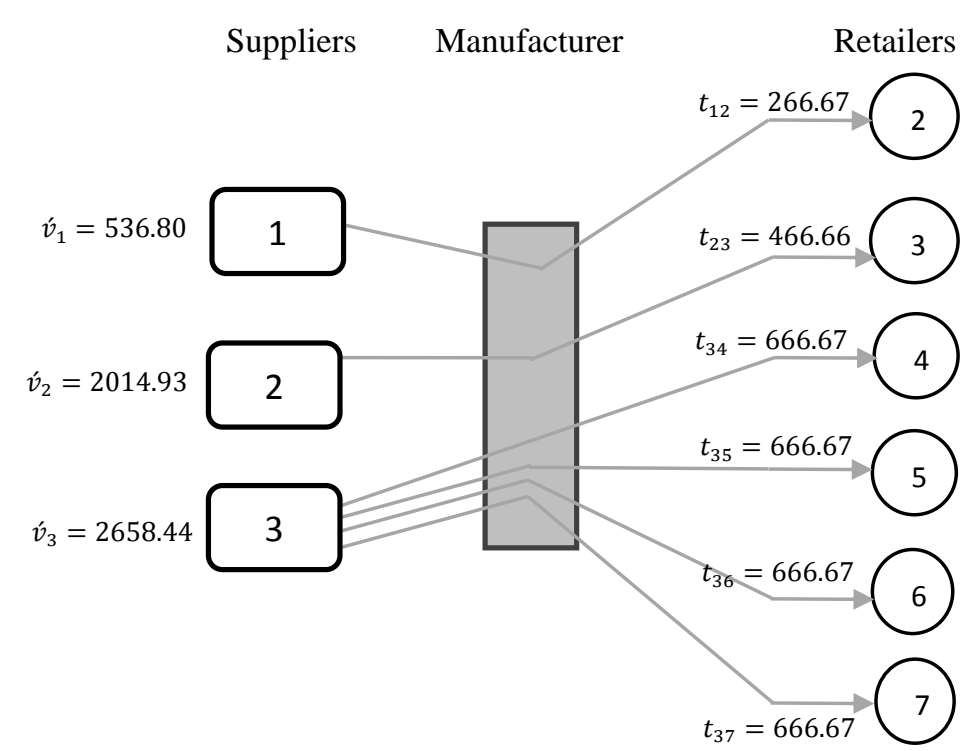

Figure 13. Network structure of SMAC according to the normal conditions (scenario 1) when no emergency stock is held.

Figure 14 represents the network flow in the third scenario in which the most cost-effective supplier is out of reach. Here, in the resilient model, the model uses the emergency stock strategy to fulfil the demand of markets 4, 5, 6, 7 (Figure 9). But in the simplified resilient model this strategy is removed. So the model has to use a more expensive strategy - extra capacity reservation at the second supplier - to fulfil the demand of these markets. Extra cost leads to higher retail prices in these markets and reduces the market share (Table 6).

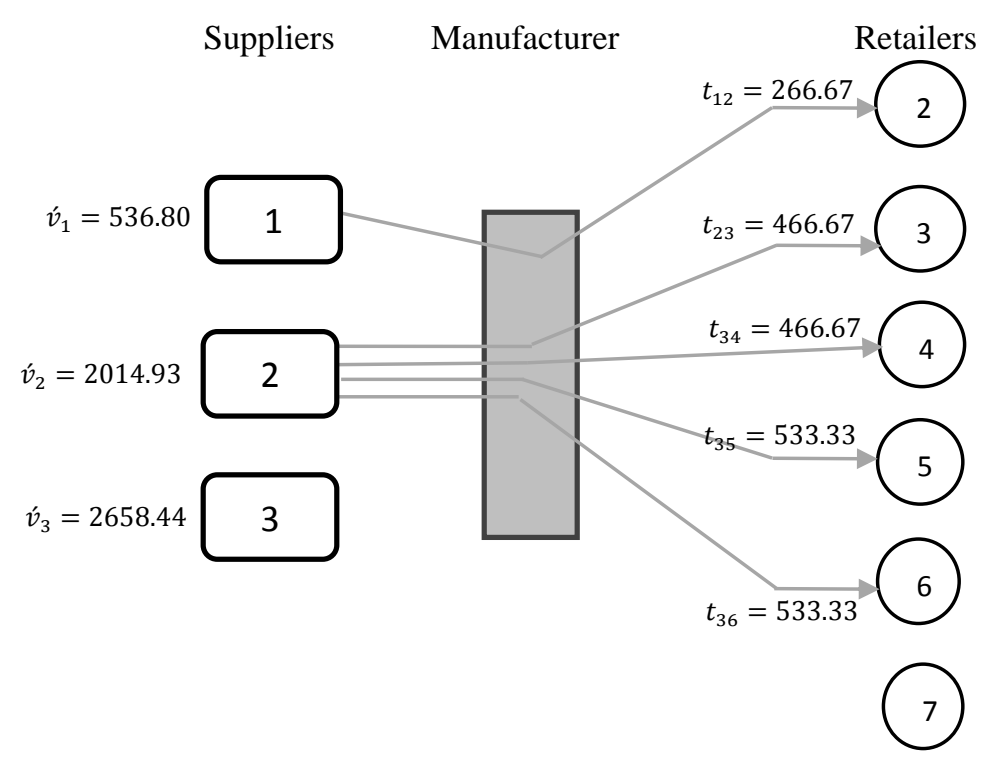

Figure 14. Network flow in scenario 3 when no emergency stock is held.

Figure 15 represents the network flow in scenario 4 in which the second and third suppliers are unavailable. In this case, there are no resources to supply the demand of the markets and therefore the demands of the fourth, fifth and sixth markets cannot be met. Consequently, this leads to a sharp drop in market shares (Table 6) and profit (Table 5). 


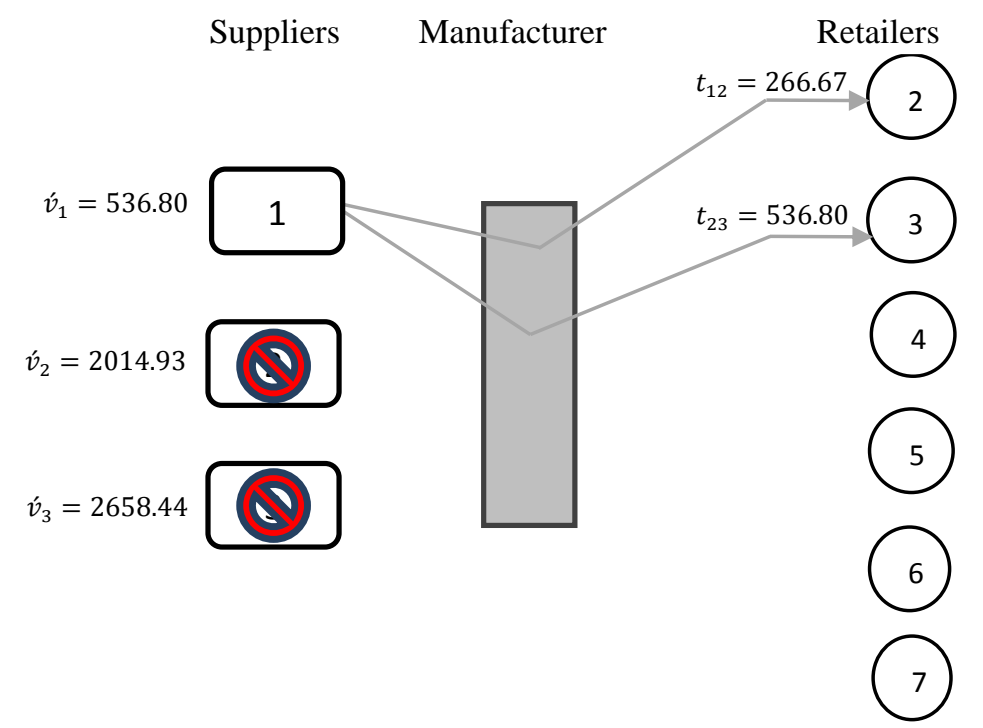

Figure 15. Network flow in scenario 4 when no emergency stock is held.

Table 6. Market share and retail prices in resilient models with and without emergency stock.

\begin{tabular}{|c|c|c|c|c|c|c|c|c|c|c|c|}
\hline \multirow[b]{2}{*}{ 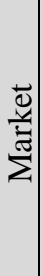 } & \multirow[b]{2}{*}{ 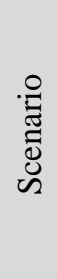 } & \multicolumn{5}{|c|}{ Resilient model with emergency stock } & \multicolumn{5}{|c|}{ Resilient model without emergency stock } \\
\hline & & Price & $\begin{array}{l}\text { Flow of } \\
\text { SMAC }\end{array}$ & 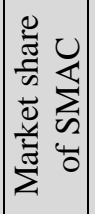 & $\begin{array}{c}\text { Flow of } \\
\text { rival }\end{array}$ & 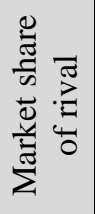 & Price & $\begin{array}{c}\text { Flow of } \\
\text { SMAC }\end{array}$ & 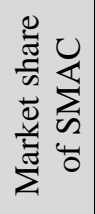 & $\begin{array}{c}\text { Flow of } \\
\text { Rival }\end{array}$ & 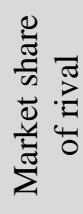 \\
\hline \multirow{4}{*}{2} & 1 & 1.850 & 266.66 & .47 & 306.66 & .53 & 1.850 & 266.66 & .47 & 306.66 & .53 \\
\hline & 2 & 1.850 & 266.66 & .47 & 306.66 & .53 & 1.850 & 266.66 & .47 & 306.66 & .53 \\
\hline & 3 & 1.850 & 266.66 & .47 & 306.66 & .53 & 1.850 & 266.66 & .47 & 306.66 & .53 \\
\hline & 4 & 1.850 & 266.66 & .47 & 306.66 & .53 & 1.850 & 266.66 & .47 & 306.66 & .53 \\
\hline \multicolumn{2}{|c|}{ Ave. } & 1.850 & 266.66 & .47 & 306.66 & .53 & 1.850 & 266.66 & .47 & 306.66 & .53 \\
\hline \multirow{4}{*}{3} & 1 & 1.783 & 466.67 & .53 & 400 & .47 & 1.783 & 466.67 & .53 & 400 & .47 \\
\hline & 2 & 1.833 & 266.67 & .40 & 400 & .60 & 1.833 & 266.67 & .40 & 400 & .60 \\
\hline & 3 & 1.783 & 466.67 & .53 & 400 & .47 & 1.783 & 466.67 & .53 & 400 & .47 \\
\hline & 4 & 1.833 & 266.67 & .40 & 400 & .60 & 1.833 & 266.67 & .40 & 400 & .60 \\
\hline \multicolumn{2}{|c|}{ Ave. } & 1.789 & 441.67 & .53 & 400 & .47 & 1.789 & 441.67 & .53 & 400 & .47 \\
\hline \multirow{4}{*}{4} & 1 & 1.733 & 666.67 & .62 & 400 & .38 & 1.733 & 666.67 & .62 & 400 & .38 \\
\hline & 2 & 1.733 & 666.67 & .62 & 400 & .38 & 1.733 & 666.67 & .62 & 400 & .38 \\
\hline & 3 & 1.733 & 666.67 & .62 & 400 & .38 & 1.783 & 466.67 & .53 & 400 & .47 \\
\hline & 4 & 1.733 & 666.67 & .62 & 400 & .38 & 1.85 & 0 & .00 & 600 & 1.0 \\
\hline \multicolumn{2}{|c|}{ Ave. } & 1.733 & 666.67 & .62 & 400 & .38 & 1.748 & 600.00 & .58 & 405 & .41 \\
\hline \multirow{4}{*}{5} & 1 & 1.733 & 666.67 & .62 & 400 & .38 & 1.733 & 666.67 & .62 & 400 & .38 \\
\hline & 2 & 1.733 & 666.67 & .62 & 400 & .38 & 1.733 & 666.67 & .62 & 400 & .38 \\
\hline & 3 & 1.733 & 666.67 & .62 & 400 & .38 & 1.766 & 533.33 & .57 & 400 & .43 \\
\hline & 4 & 1.733 & 666.67 & .62 & 400 & .38 & 1.850 & 0 & .00 & 600 & 1.0 \\
\hline \multicolumn{2}{|c|}{ Ave. } & 1.733 & 666.67 & .62 & 400 & .38 & 1.744 & 616.66 & .59 & 405 & .40 \\
\hline \multirow{4}{*}{6} & 1 & 1.733 & 666.67 & .62 & 400 & .38 & 1.733 & 666.67 & .62 & 400 & .38 \\
\hline & 2 & 1.733 & 666.67 & .62 & 400 & .38 & 1.733 & 666.67 & .62 & 400 & .38 \\
\hline & 3 & 1.733 & 666.67 & .62 & 400 & .38 & 1.766 & 533.33 & .57 & 400 & .43 \\
\hline & 4 & 1.733 & 666.67 & .62 & 400 & .38 & 1.85 & 0 & .00 & 600 & 1.0 \\
\hline \multicolumn{2}{|c|}{ Ave. } & 1.733 & 666.67 & .62 & 400 & .38 & 1.744 & 616.66 & .59 & 405 & .40 \\
\hline \multirow{4}{*}{7} & 1 & 1.683 & 666.67 & .53 & 600 & .47 & 1.683 & 666.67 & .53 & 600 & .47 \\
\hline & 2 & 1.683 & 666.67 & .53 & 600 & .47 & 1.683 & 666.67 & .53 & 600 & .47 \\
\hline & 3 & 1.683 & 666.67 & .53 & 600 & .47 & 1.775 & 0 & .00 & 900 & 1.0 \\
\hline & 4 & 1.683 & 666.67 & .53 & 600 & .47 & 1.775 & 0 & .00 & 900 & 1.0 \\
\hline \multicolumn{2}{|c|}{ Ave. } & 1.683 & 666.67 & .53 & 600 & .47 & 1.708 & 483.33 & .38 & 537.5 & .62 \\
\hline
\end{tabular}




\section{Contribution of Risk Mitigation Strategies to the Price Stability in the Markets}

In Table 6, equilibrium prices in the markets and the market shares in the resilient model with no emergency stock compared with those in the resilient model with emergency stock are summarized. As intuition suggests, not having emergency stock leads to reduction in the SC's supply capability and increases the rivals' market share.

Risk mitigation strategies enables the SC to provide a steady flow of product to the markets in all situations. Total product supply to each market is the critical factor determining the equilibrium product price in that market. Therefore risk mitigation strategies have a key role in stabilizing prices in the markets. In this section, we use the results summarized in Tables 4 and 6 to analyze the contributions of risk mitigation strategies in stabilizing market prices. Figure 17 displays the equilibrium market price dispersion in four scenarios and three cases: i) without risk mitigation strategies; ii) with only "extra capacity" strategy; and iii) with both "extra capacity" and "emergency stock" strategies.

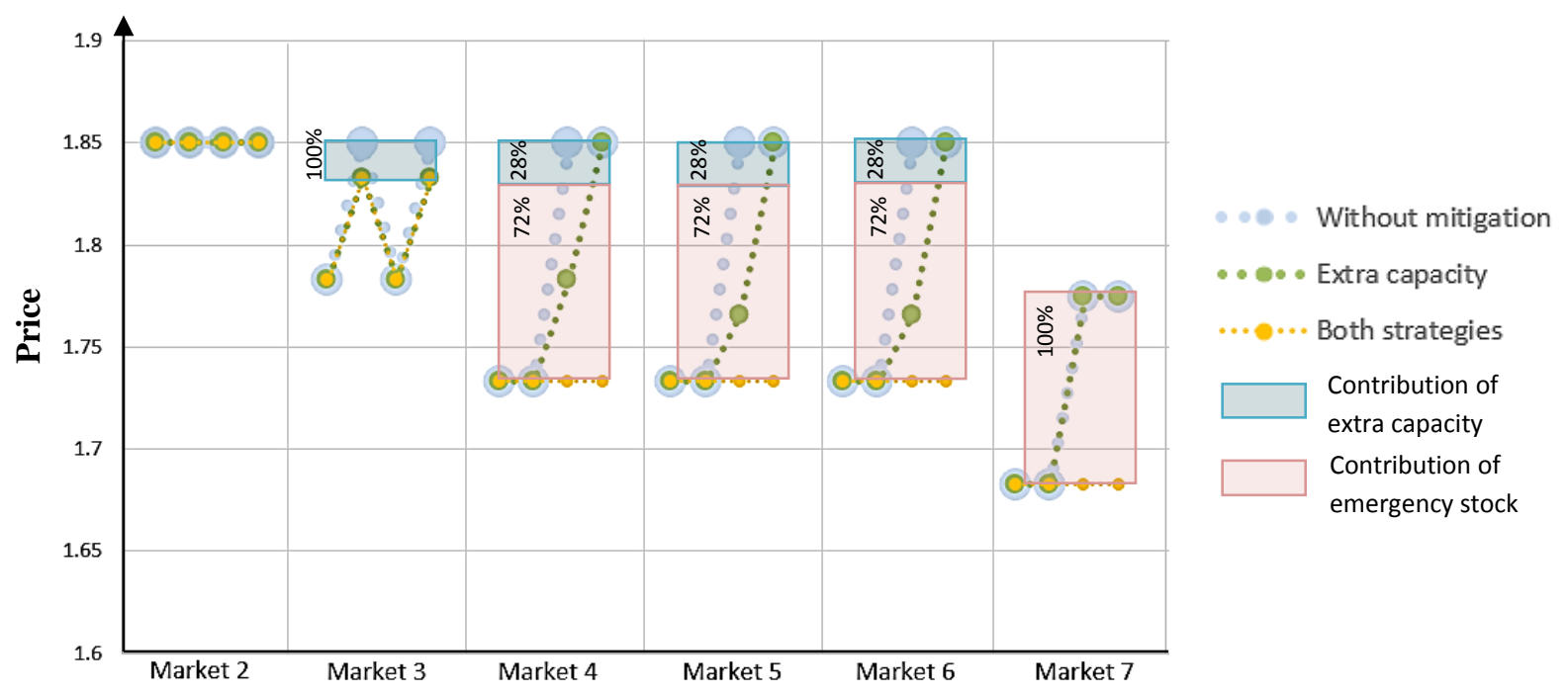

Figure 17. Contribution of risk mitigation strategies in stabilizing markets' prices.

Based on these results, we conclude that:

- Adding "extra capacity" strategy, decreases price dispersion 0 percent in the second and seventh market, 100 percent in the third market, 28 percent in the fourth, fifth and sixth markets. Therefore, this strategy can stabilize markets' prices up to 30.7 percent in average.

- After adding "emergency stock" strategy to the SC with "extra capacity" strategy, price dispersion in the markets reduces 0 percent in the second and third markets, 72 percent in the fourth, fifth and sixth markets, and 100 percent in the seventh market. So this strategy helps to stabilize the market prices up to 52.7 percent in average.

- If both risk mitigation strategies are incorporated in the $\mathrm{SC}$, then the price stability is 3.7 times higher than stability in the markets of the SC without mitigation strategies. In this market stabilization, the contribution of "emergency stock" strategy (72 percent) is 2.57 times as much as that of "extra capacity" (28 percent).

\section{Contributions of Risk Mitigation Strategies to the Stability of the market shares}

Risk mitigation strategies increase the product supply capability and help it to retain its shares in the markets in 
all situations. This constant presence in the markets even in the event of disruptions stabilizes the position of SC, helps it to keep its customers and make long-term relationships with them, and improves its brand reputation. All these characteristic positively affect the profitability of the SC in the long term. In this section, we use the results summarized in Tables 4 and 6 to analyze the contributions of risk mitigation strategies in stabilizing SC market shares. Figure 18 displays the SC market shares in four scenarios in three cases: i) without risk mitigation strategies; ii) with only "extra capacity" strategy; and iii) with both "extra capacity" and "emergency stock" strategies.

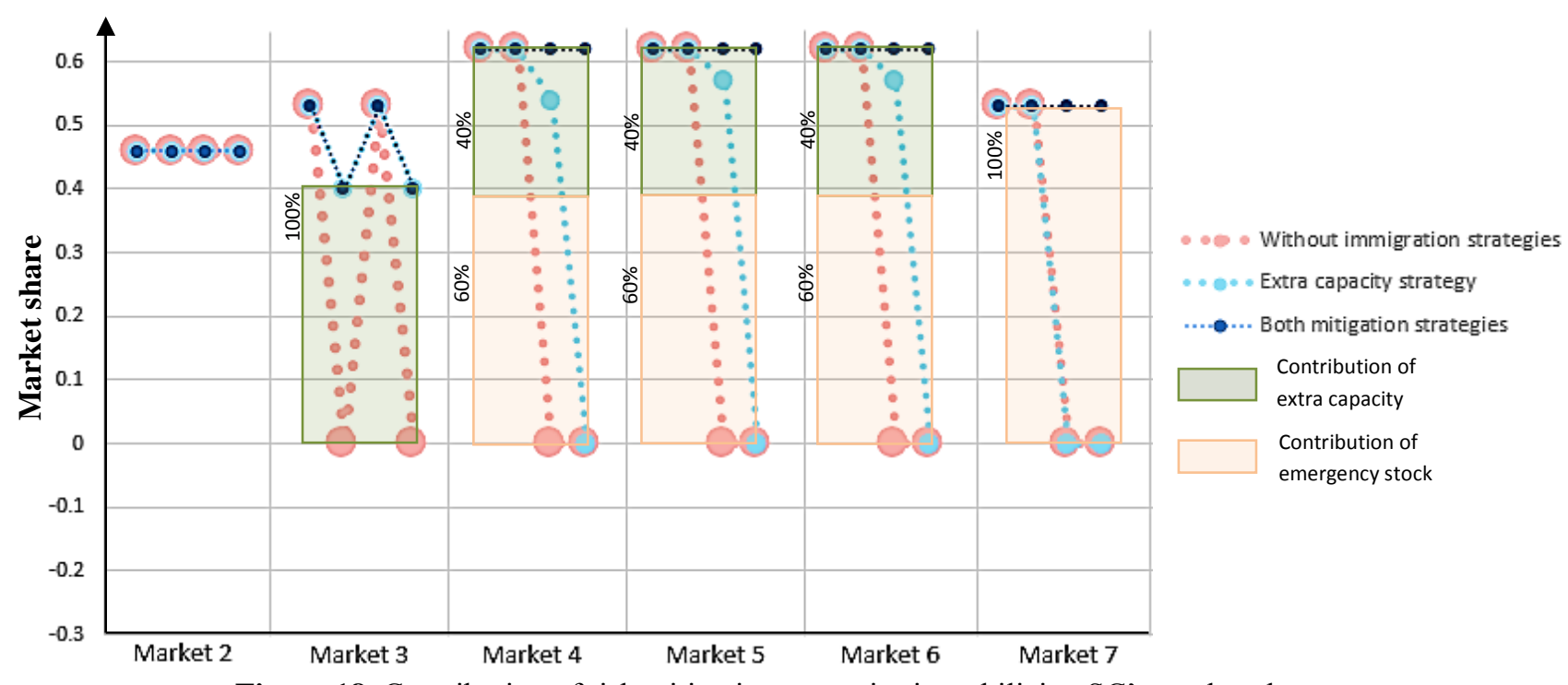

Figure 18. Contribution of risk mitigation strategies in stabilizing SC's market shares.

Based on these results, we conclude that:

- Adding "extra capacity" strategy decreases market share variation 0 percent in the second and seventh market, 100 percent in the third market, 40 percent in the fourth, fifth, and sixth markets. Thus this strategy can stabilize the position of the SC in the markets 36.1 percent in average.

- After adding "emergency stock" strategy to the SC with "extra capacity" strategy, the market share dispersion reduces 0 percent in the second and third markets, 60 percent in the fourth, fifth, and sixth markets, and 100 percent in the seventh markets. Therefore, this strategy helps to stabilize the SC share in the markets about 47.1 percent in average.

- The market share stability with both risk mitigation strategies is 13.5 times higher than that of the model without mitigation strategies. In this market share retention, the contribution of "emergency stock" strategy is 1.5 times as much as that of "extra capacity" in most of the markets.

\section{Prioritization of the Risk Mitigation Strategies}

In the scenarios and in the markets in which both risk mitigation strategies are applicable, their priority in selection depends on their costs (unit inventory holding cost for "emergency stock" strategy and cost of unit capacity for "extra capacity" strategy). To determine this dependency, we solve the model for different values of inventory holding cost and capacity cost. The results of strategy selection are displayed in Figure 19. Based on these results, the space representing (inventory cost, capacity cost) combinations can be divided into three regions:

- $\underline{\text { Region I: }}$ In this region, the inventory cost is significantly higher than the capacity cost (in the case 
problem inventory cost $\gtrsim 0.052+0.8 \times$ capacity cost $)$. Therefore, only "extra capacity" risk mitigation strategy is selected by the model to counter the negative impacts of disruptions.

- Region II: In this region, the capacity cost is significantly higher than the inventory cost (in the case problem inventory cost $\lesssim 0.01+0.75 \times$ capacity cost). Therefore, only "emergency stock" risk mitigation strategy is selected by the model to counter the negative impacts of disruptions.

- Region III: In this region, the model decides to have a combination of both policies. Therefore, the network keeps emergency stock besides reserving extra capacity to counter the negative impacts of disruption.

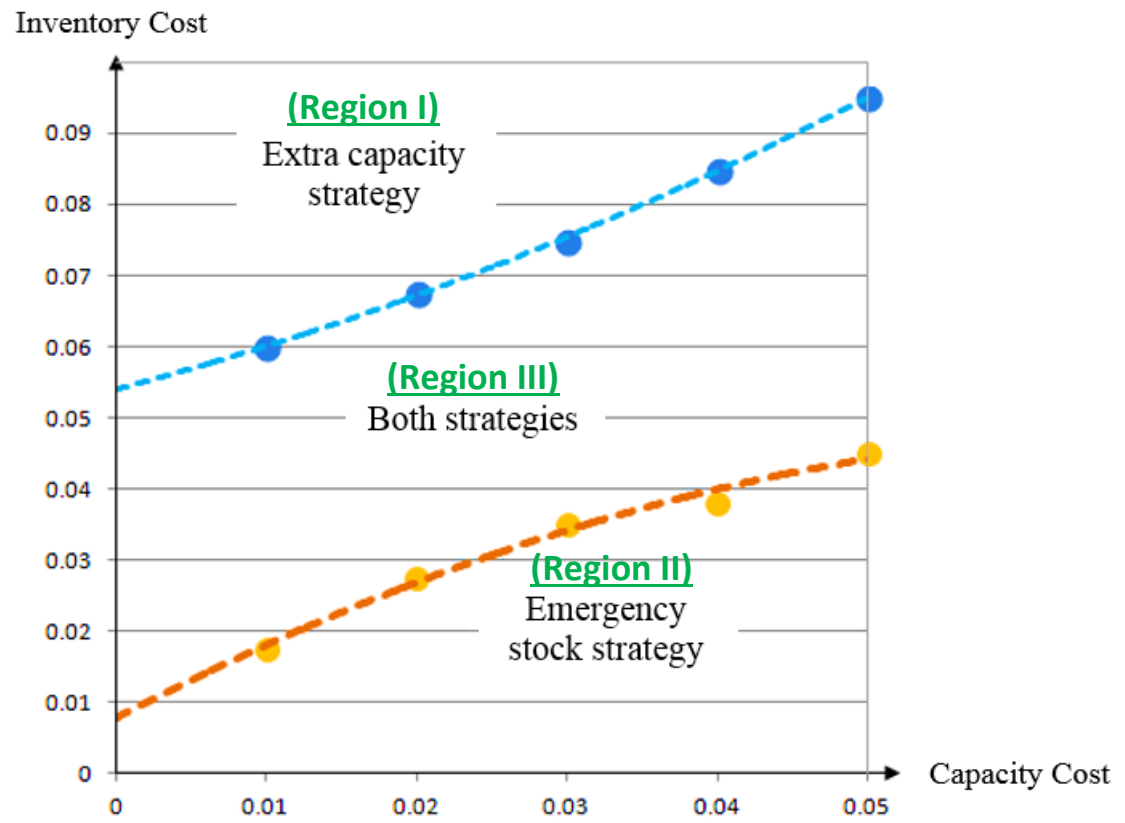

Figure 19. Prioritization of risk mitigation strategies with respect to different inventory and capacity costs.

Generalizing the outputs of the case problem, leads to the following insights:

- If all the potential supply paths ending at a competitive market are unreliable, then holding downstream "emergency stock" is the most preferable risk mitigation strategy. Keeping emergency stock becomes more appealing as the number of target markets increases.

- Moreover, if at least one reliable path exists among the potential supply paths servicing a competitive market, then the most preferable risk mitigation strategy is the most economic (least costly) one. Increasing the number of target markets, further highlights the importance of the most economic risk management strategy.

\section{CONCLUSION}

This paper aims to address the design of a resilient SC network that is susceptible to two major risks namely a tough competition in the market and unreliability of suppliers. There are several practical cases in the literature indicating that in today's highly competitive markets any failure to fulfil customer demand is not tolerated and results in loss of market share. However, with globalization, the number of suppliers has increased dramatically leading to a far more complex environment and the chances that suppliers will face disruption have also risen. Therefore, companies need to plan ahead and consider what risk mitigation strategies they can use if a supplier experiences disruption. Using these mitigation strategies firms or the SCs can recover quickly from such 
disruption. To this end, the notion of a resilient SC has been developed. SC resilience is built on the idea that firms need to create redundancy and flexibility that can be brought into play if a disruption occurs. Several risk mitigation strategies are proposed, and the most popular ones in practice include keeping extra emergency stock, reserving extra capacity at suppliers, and multiple-sourcing.

In this paper, we incorporate these strategies in the design stage of the SC and investigate how these policies work to the advantage of SCs. Firms might be reluctant to implement these policies since they are considered costly. In this paper, we show that such policies, even though costly, help to ensure that companies can maintain their market share even when faced with disruption. What is also noteworthy is that such strategies not only work to the advantage of the company but their customers also benefit from them. Thoughtfully incorporating such risk mitigation policies results in price reduction that customers can enjoy. This is mainly because, with these strategies in place, SCs can avoid product scarcity and can keep on competing with rivals if disruption occurs.

Moreover, using a controlled experimental setting through studying the impact of risk mitigation strategies and cost parameters, we are able to draw insights on the performance of these risk strategies. We are able to identify under different structures how these strategies perform with respect to profit, stability of the market price and also market share. Our findings show that creating resiliency boosts average supply capability, profit, and market share of SCs. However, the cost of implementing mitigation strategies may lead to profit reduction if no disruption occurs. The model we developed in this paper captures this trade-off and helps to compromise these two aspects. Also we show that resilience of SCs stabilizes retail prices in competitive markets and reduces their fluctuations in the event of disruptions. The contribution of risk mitigation strategies, "keeping strategic stock" and "reserving extra capacity", in the stabilizations of a SC's market share and markets' prices depends on the: I) SC's network structure and II) cost components of the risk mitigation strategies. If all the potential supply paths of the SC ending at a competitive market are unreliable, then holding downstream "emergency stock" is the most preferable risk mitigation strategy. Moreover, if at least one reliable path exists among the potential supply paths servicing a competitive market, then the most preferable risk mitigation strategy is the most economic (least costly) one.

\section{APPENDIX A}

Proof of Lemma 1: First assume that there are $N$ rivals competing in a market, $m$. Production price for each rival is $c_{i},(i=1,2, \ldots, N)$. Production quantity of each rival is $q_{i}$ and retail price of product in the market is $p_{m}$. Thus the profit for rival $i$ is

$$
\pi_{i}=\left(p_{m}-c_{i}\right) \cdot q_{i}=\left(a_{m}-b_{m} \cdot\left(q_{i}+\sum_{\substack{j=1 \\ j \neq i}}^{N} q_{j}\right)-c_{i}\right) \cdot q_{i}
$$

We differentiate this profit function over production quantity $q_{i}$. Using the first order condition, we have:

$$
\frac{\partial \pi_{i}}{\partial q_{i}}=\left[a_{m}-b_{m} \cdot\left(\sum_{\substack{j=1 \\ j \neq i}}^{N} q_{j}\right)-c_{i}\right]-2 \cdot b_{m} \cdot q_{i}
$$

Additionally, the second order condition of this function is 


$$
\frac{\partial^{2} \pi_{i}}{\partial q_{i}^{2}}=-2 . b_{m}<0
$$

It is obvious that profit function has a unique optimal solution shown as $\tilde{q}_{i}$. If we set the first order derivatives to zero we have

$$
\frac{1}{2} . \tilde{q}_{i}=\frac{a_{m}-c_{i}}{2 . b_{m}}-\frac{1}{2} \cdot \sum_{j=1}^{N} \tilde{q}_{j} \quad i=1,2, \ldots, N
$$

If we add up these $N$ equalities we have

$$
\tilde{q}_{i}=\frac{a_{m}-c_{i}}{b_{m} \cdot(N+1)}
$$

Substituting $\tilde{q}_{i}$ into (8) we have

$$
\tilde{p}=\frac{a_{m}+\sum_{i=1}^{N} c_{i}}{N+1}
$$

$N$ in the denominator of (38) and (39) represents the number of rivals in that market. In our problem one of these rivals is the new chain for which production cost is $\sum_{t=1}^{\left|T^{(m)}\right|} c_{t} x_{t}^{n}$ (based on Equation 9). In our problem the number of each market's rivals is equal to $N=\left|R^{(m)}\right|+w_{m}$. Substituting these equations in (38) results (10). By following the same approach we can easily derive Equation (11) from Equation (39).

\section{APPENDIX B}

Notice that non-linear revenue and product flow occur in the retailers at which two or more paths end. If only one path ends at the retailer then the variable governing the assignment of flow to the path, $\boldsymbol{x}_{\boldsymbol{t}}^{\boldsymbol{s}}$, converts to a binary variable that takes 1 when the path is active and 0 otherwise. Linearization of the multiplication of two binary variables is not a cumbersome task. The situation becomes problematic when more than two paths end at a retailer and it needs to be decided how to divide the order quantity originating from the retailer among these paths. In other words, the problem occurs when the variable $\boldsymbol{x}_{\boldsymbol{r}}^{\boldsymbol{s}}$ corresponding to these paths can take any value in $[0,1]$. Multiplying two continuous variables leads to non-linear terms in the objective function and constraints. To clarify the issue further, consider a retailer $m$ that lies at the end of two paths. The unit production and distribution costs in the first and second paths are 1.65 and 1.5 , respectively. In this case the profit generated by this retailer in a given scenario, $s$, can be formulated as follows:

$$
\begin{aligned}
\left(\frac{a_{m}+\sum_{r=1}^{\left|R^{(m)}\right|} c_{r}+\left(1.65 x_{1}^{S}+1.5 x_{2}^{S}\right)}{\left(2+\left|R^{(m)}\right|\right)}-1.65\right)\left(\frac{a_{m}-\left(1.65 x_{1}^{S}+1.5 x_{2}^{S}\right)}{b_{m}\left(2+\left|R^{(m)}\right|\right)}\right) x_{1}^{S}+ \\
\left(\frac{a_{m}+\sum_{r=1}^{R^{(m)} \mid} c_{r}+\left(1.65 x_{1}^{S}+1.5 x_{2}^{S}\right)}{\left(2+\left|R^{(m)}\right|\right)}-1.50\right)\left(\frac{a_{m}-\left(1.65 x_{1}^{S}+1.5 x_{2}^{S}\right)}{b_{m}\left(2+\left|R^{(m)}\right|\right)}\right) x_{2}^{S}
\end{aligned}
$$

This function is depicted in Figure 3 with respect to $x_{1}^{S}\left(x_{2}^{S}=1-x_{1}^{S}\right)$. As observed, the captured profit is a strictly decreasing function. In our numerical analysis we observe that the profit function is either slightly concave or slightly convex. This specification of profit function derives us to substitute it with a piecewise linear function. If we break the range $[0,1]$ into a number of sections - say three - the resulting three-piece linear function, an approximation of (40), would correspond to Equation 41 (Figure 3). Increasing the number of parts in the linear 
function improves its approximation. But dividing the range should be discontinued once the approximation seems acceptable.

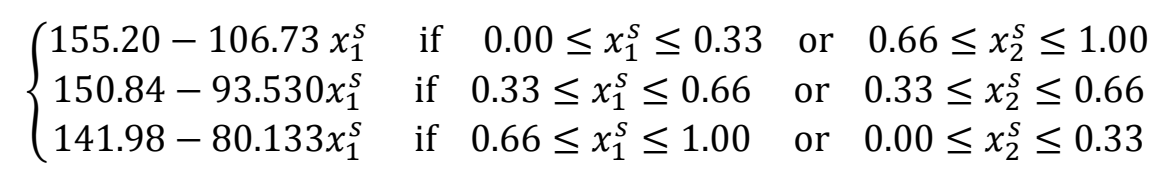

A similar approach can be used to linearize the assigned equilibrium flow of the paths ending at the investigated retailer (Figure 4 and Equations 42 and 43).

$$
\begin{gathered}
\left(\frac{a_{m}-\left(1.65 x_{1}^{S}+1.5 x_{2}^{S}\right)}{b\left(2+\left|R^{(m)}\right|\right)}\right) x_{1}^{S}=\left\{\begin{array}{l}
3.520+600.66 x_{1}^{S} \text { if }\left(0.00 \leq x_{1}^{S} \leq 0.33 \text { or } 0.66 \leq x_{2}^{S} \leq 1.00\right) \\
47.08+468.66 x_{1}^{S} \text { if }\left(0.33 \leq x_{1}^{S} \leq 0.66 \text { or } 0.33 \leq x_{2}^{S} \leq 0.66\right) \\
135.47+334.6 x_{1}^{S} \text { if }\left(0.66 \leq x_{1}^{S} \leq 1.00 \text { or } 0.00 \leq x_{2}^{S} \leq 0.33\right)
\end{array}\right. \\
\left(\frac{a_{m}-\left(1.65 x_{1}^{S}+1.5 x_{2}^{S}\right)}{b\left(2+\left|R^{(m)}\right|\right)}\right) x_{2}^{S}=\left\{\begin{array}{l}
663.1-800.66 x_{1}^{S} \text { if } 0.00 \leq x_{1}^{S} \leq 0.33 \text { or } 0.66 \leq x_{2}^{S} \leq 1.00 \\
619.5-668.66 x_{1}^{S} \text { if } 0.33 \leq x_{1}^{S} \leq 0.66 \text { or } 0.33 \leq x_{2}^{S} \leq 0.66 \\
530.9-534.66 x_{1}^{S} \text { if } 0.66 \leq x_{1}^{S} \leq 1.00 \text { or } 0.00 \leq x_{2}^{S} \leq 0.33
\end{array}\right.
\end{gathered}
$$

After piecewise linearization, an approximation of equilibrium flow function corresponds to:

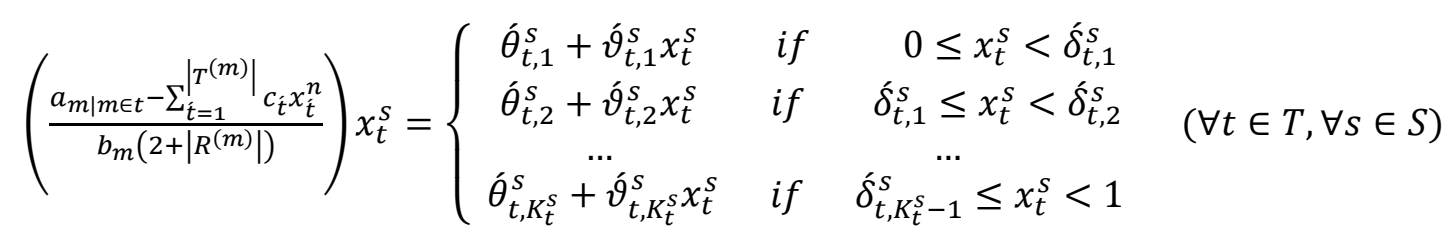

\section{APPENDIX C}

We describe how the multi-part linear functions developed in Section 3.4 can be substituted into the proposed model (22-32) to transform it into a mixed integer linear (MIL) mathematical model. Existing commercial optimization software such as LINGO, CPLEX, and GAMS can be used to solve the linearized model. In order to substitute these functions in the model, we need to define some new binary variables that determine which part of the substituted multi-part profit and flow functions should be considered. $\alpha_{t, k}^{S}$ and $\alpha_{t, k}^{S}$ are binary variables that are equal to 1 if variable $x_{t}^{S}$ accepts a value in part $k\left(k=1,2, \ldots, K_{t}^{S}\right)$ of its piecewise linearized profit and flow function respectively; 0 otherwise. Notice that $\sum_{k=1}^{K_{t}^{S}} \alpha_{t, k}^{S}=w_{m}$ and $\sum_{k=1}^{K_{t}^{S}} \alpha_{t, k}^{S}=w_{m}(\forall t \in$ $\left.T^{(m)}, \forall m \in M\right)$. Using these newly defined variables, the following term is substituted in place of the nonlinear profit term in the objective function (22):

$$
\left(\frac{a_{m \mid m \in t}+\sum_{r=1}^{\left|R^{(m)}\right|} c_{r}+\sum_{t=1}^{T^{(m)} \mid} c_{t} x_{t}^{n}}{\left(2+\left|R^{(m)}\right|\right)}-c_{t}\right)\left(\frac{a_{m}-\sum_{t=1}^{T^{(m)} \mid} c_{t} x_{t}^{n}}{b_{m}\left(2+\left|R^{(m)}\right|\right)}\right) x_{t}^{S}=\sum_{k=1}^{K_{t}^{S}} \alpha_{t, k}^{S}\left(\theta_{t, k}^{S}+\vartheta_{t, k}^{S} x_{t}^{S}\right),(\forall t \in T, \forall s \in S)
$$

Where

$$
\sum_{k=1}^{K_{t}^{S}} \alpha_{t, k}^{S} \delta_{t, k-1}^{S} \leq x_{t}^{S} \leq \sum_{k=1}^{K_{t}^{S}} \alpha_{t, k}^{S} \delta_{t, k}^{S}
$$

$(\forall t \in T, \forall s \in S)$

Nonlinear equilibrium flow function in Constraints (23) and (24) will be substituted by the following terms:

$$
\left(\frac{a_{m}-\sum_{t=1}^{\left|T^{(m)}\right|} c_{t} x_{t}^{n}}{b_{m}\left(2+\left|R^{(m)}\right|\right)}\right) x_{t}^{S}=\sum_{k=1}^{K_{t}^{S}} \dot{\alpha}_{t, k}^{S}\left(\dot{\theta}_{t, k}^{s}+\hat{\vartheta}_{t, k}^{s} x_{t}^{S}\right)
$$

$(\forall t \in T, \forall s \in S)$

Where 


$$
\sum_{k=1}^{K_{t}^{S}} \dot{\alpha}_{t, k}^{S} \dot{\delta}_{t, k-1}^{s} \leq x_{t}^{S} \leq \sum_{k=1}^{K_{t}^{s}} \dot{\alpha}_{t, k}^{S} \dot{\delta}_{t, k}^{s}
$$

$(\forall t \in T, \forall s \in S)$

Linearization of the multiplication of a binary and a continuous variable in (35) and (38) is straightforward.

\section{ACKNOWLEDGMENT}

We would like to thank Prof. Ruud Teunter and two anonymous referees who helped us improve the paper substantially with their constructive comments.

\section{REFERENCES}

Baghalian A., Rezapour S., Farahani RZ. (2013) Robust supply chain network design with service level against disruptions and demand uncertainties: A real-life case. European Journal of Operational Research 227(1); 199-215.

Boyaci T., Gallego G. (2004) Supply chain coordination in a market with customer service competition. Production and Operations Management 13(1); 3-22.

Chopra S., Reinhardt G., Mohan U. (2007) The importance of decoupling recurrent and disruption risks in a supply chain. Naval Research Logistics, 54(5); 544-555.

Chopra S., Sodhi MS. (2004) Managing risk to avoid supply chain breakdown, MIT Sloan Management Review 46(1); 53-62.

Dada M., Petruzzi NC., Schwarz LB. (2007) A newsvendor's procurement problem when suppliers are unreliable. Manufacturing \& Service Operations Management, 9(1); 9-32.

Fahimnia, B., Tang, CS., Davarzani, H., Sarkis, J. (2015) Quantitative models for managing supply chain risks: A review. European Journal of Operational Research, 247(1); 1-15.

Farahani, RZ., Rezapour, S., Drezner, T., Fallah, S. (2014) Competitive supply chain network design: An overview of classifications, models, solution techniques and applications. Omega, 45(1); 92-118.

Feng, Q, Shi, R. (2012) Sourcing from multiple suppliers for price-dependent demands. Production and Operations Management 21(3) 547-563.

Goh, M., Lim, JY., Meng, F. (2007). A stochastic model for risk management in global supply chain networks. European Journal of Operational Research, 182(1); 164-173.

Ha, AY., Tong, S. (2008) Contracting and information sharing under supply chain competition. Management Science 54(4); 701-715.

Hendricks, KB., Singhal, VR. (2005) Association between supply chain glitches and operating performance. Management Science 51(5); 695-711.

Huang, E. and Goetschalckx, M. (2014). Strategic robust supply chain design based on the Pareto-optimal tradeoff between efficiency and risk. European Journal of Operational Research, 237(2), 508-518.

Ivanov, D., Sokolov, B., Dolgui, A. (2014a) The ripple effect in supply chains: Trade-off 'efficiency flexibilityresilience' in disruption management. International Journal of Production Research, 52(7), 2154-2172.

Ivanov, D., Sokolov, B., Pavlov, A. (2014b) Optimal distribution (re)planning in a centralized multistage network under conditions of ripple effect and structure dynamics. European Journal of Operational Research, 237(2), 758-770.

Khan M., Jaber MY., Ahmad A-R. (2014) An integrated supply chain model with errors in quality inspection and learning in production. Omega 42(1); 16-24.

Kleindorfer, PR., Saad, GH. (2005). Managing disruption risks in supply chains. Production and operations management, 14(1); 53-68.

Klibi, W., Martel, A., Guitouni, A. (2010). The design of robust value-creating supply chain networks: a critical review. European Journal of Operational Research, 203(2); 283-293.

Klibi, W., Martel, A. (2012) Modeling approaches for the design of resilient supply networks under disruptions, International Journal of Production Economics, 135(2); 882-898.

Lewis BM., Erera AL., Nowak MA., White CC. (2013) Managing inventory in global supply chains facing port-of-entry disruption risks. Transportation Science 47(2); 162-180.

Lin CC., Wang TH. (2011) Build-to-order supply chain network design under supply and demand 
uncertainties. Transportation Research: Part B 45(8); 1-15.

Martha J., Subbakrishna S. (2002) Targeting a just-in-case supply chain for the inevitable next disaster. Supply Chain Management Review 6(5); 18-23.

Majumder, P., Srinivasan, A. (2008) Leadership and competition in network supply chains. Management Science 54(6); 1189-1204.

Narayanan, VG., Raman, A., Singh, J. (2005) Agency costs in a supply chain with demand uncertainty and price competition. Management Science 51(1); 120-132.

Qi L., Z.-J., Shen, M., Snyder, LV. (2010) The effect of supply disruptions on supply chain design decisions. Transportation Science 44(2); 274-289.

Schmidt, W., Simchi- Levi, D. (2013) Nissan Motor Company Ltd: Building operational resiliency, MIT Sloan Management, Case Number 13-150.

Sharma A., Banerjee S. (2013) Optimal price markup policy for an inventory model with random price fluctuations and option for additional purchase. International Journal of Production Economics 146(2); 620 633.

Pickett CB. (2003) Strategies for maximizing supply chain resilience: learning from the past to prepare for the future. Thesis. Massachusetts Institute of Technology.

Rezapour S., Hassani A., Farahani RZ. (2015) Concurrent design of product family and supply chain network considering quality and price. Transportation Research Part E 81(1); 18-35.

Schütz P., Tomasgard A., Ahmed S. (2009) Supply chain design under uncertainty using sample average approximation and dual decomposition. European Journal of Operational Research 199(1); 409-419.

Sheffi Y. (2007) The resilient enterprise: overcoming vulnerability for competitive advantage. MIT Press. Cambridge, MA.

Shen ZJ., Daskin M. (2005) Trade-offs between customer service and cost in integrated supply chain design. Manufacturing \& Service Operations Management 7(3); 188-207.

Tang, C., \& Tomlin, B. (2008). The power of flexibility for mitigating supply chain risks. International Journal of Production Economics, 116(1); 12-27.

Tian, J., Yue, J. (2014) Bounds of Relative Regret Limit in p-Robust Supply Chain Network Design. Production and Operations Management. 23(10); 1811-1831.

Tomlin B. (2006) On the value of mitigation and contingency strategies for managing supply chain disruption risks. Management Science. 52(5); 639-657.

Wilson MC. (2007) The impact of transportation disruptions on supply chain performance. Transportation Research Part E 43(4); 295-320.

Xiao, T., Yang, D. (2008). Price and service competition of supply chains with risk-averse retailers under demand uncertainty. International Journal of Production Economics, 114(1); 187-200.

Zhang, D. (2006). A network economic model for supply chain versus supply chain competition. Omega, 34(3), 283-295.

Zhao, X. (2008) Coordinating a supply chain system with retailers under both price and inventory competition. Production and Operations Management 17(5); 532-542. 